\title{
Nonlinear transient amplification in recurrent neural networks with short-term plasticity
}

\author{
Yue Kris $\mathrm{Wu}^{1,2}$ and Friedemann Zenke ${ }^{1, *}$ \\ ${ }^{1}$ Friedrich Miescher Institute for Biomedical Research, 4058 Basel, Switzerland \\ ${ }^{2}$ Faculty of Natural Sciences, University of Basel, 4033 Basel, Switzerland \\ *Correspondence: friedemann.zenke@fmi.ch
}

\begin{abstract}
To rapidly process information, neural circuits have to amplify specific activity patterns transiently. How the brain performs this nonlinear operation remains elusive. Hebbian assemblies are one possibility whereby symmetric excitatory connections boost neuronal activity. However, such Hebbian amplification is often associated with dynamical slowing of network dynamics, non-transient attractor states, and pathological run-away activity. Feedback inhibition can alleviate these effects but typically linearizes responses and reduces amplification gain. At the same time, other alternative mechanisms rely on asymmetric connectivity, in conflict with the Hebbian doctrine. Here we propose nonlinear transient amplification (NTA), a plausible circuit mechanism that reconciles symmetric connectivity with rapid amplification while avoiding the above issues. NTA has two distinct temporal phases. Initially, positive feedback excitation selectively amplifies inputs that exceed a critical threshold. Subsequently, short-term plasticity quenches the run-away dynamics into an inhibition-stabilized network state. By characterizing NTA in supralinear network models, we establish that the resulting onset transients are stimulus selective and well-suited for speedy information processing. Further, we find that excitatory-inhibitory co-tuning widens the parameter regime in which NTA is possible. In summary, NTA provides a parsimonious explanation for how excitatory-inhibitory co-tuning and short-term plasticity collaborate in recurrent networks to achieve transient amplification.
\end{abstract}

\section{Introduction}

Perception in the brain is reliable and strikingly fast. Recognizing a familiar face or locating an animal in a picture only takes a split second (Thorpe et al., 1996). This pace of processing is truly remarkable since it involves several recurrently connected brain areas each of which has to selectively amplify or suppress specific signals before propagating them further. This processing is mediated through circuits with several intriguing properties. First, excitatory-inhibitory (EI) currents into individual neurons are commonly correlated in time and co-tuned in stimulus space 
(Wehr and Zador, 2003; Froemke et al., 2007; Okun and Lampl, 2008; Hennequin et al., 2017; Rupprecht and Friedrich, 2018; Znamenskiy et al., 2018). Second, neural responses to stimulation are shaped through diverse forms of short-term plasticity (STP) (Tsodyks and Markram, 1997; Markram et al., 1998; Zucker and Regehr, 2002; Pala and Petersen, 2015). Finally, mounting evidence suggests that amplification rests on neuronal ensembles with strong recurrent excitation (Marshel et al., 2019; Peron et al., 2020), whereby excitatory neurons with similar tuning preferentially form reciprocal connections (Ko et al., 2011; Cossell et al., 2015). Such predominantly symmetric connectivity between excitatory cells is consistent with the notion of Hebbian cell assemblies (Hebb, 1949), which are considered an essential component of neural circuits and the putative basis of associative memory (Harris, 2005; Josselyn and Tonegawa, 2020). Computationally, Hebbian cell assemblies can amplify specific activity patterns through positive feedback, also referred to as Hebbian amplification. Based on these principles, several studies have shown that Hebbian amplification can drive persistent activity that outlasts a preceding stimulus (Hopfield, 1982; Amit and Brunel, 1997; Yakovlev et al., 1998; Wong and Wang, 2006; Zenke et al., 2015; Gillary et al., 2017), comparable to selective delay activity observed in the prefrontal cortex when animals are engaged in working memory tasks (Funahashi et al., 1989; Romo et al., 1999).

However, in most brain areas, evoked responses are transient and sensory neurons typically exhibit pronounced stimulus onset responses, after which the circuit dynamics settle into a lowactivity steady-state even when the stimulus is still present (DeWeese et al., 2003; Mazor and Laurent, 2005; Bolding and Franks, 2018). Preventing run-away excitation and multi-stable attractor dynamics in recurrent networks requires powerful and often finely tuned feedback inhibition resulting in El balance (Amit and Brunel, 1997; Compte et al., 2000; Litwin-Kumar and Doiron, 2012; Ponce-Alvarez et al., 2013; Mazzucato et al., 2019). However, feedback inhibition tends to linearize steady-state activity (Van Vreeswijk and Sompolinsky, 1996; Baker et al., 2020) and does not necessarily generate pronounced onset responses consistent with experiments. While feedforward inhibition provides one possible explanation for transient onset dynamics (Wehr and Zador, 2003; Vogels et al., 2011; Gjoni et al., 2018), it does not explain the recurrent excitation commonly seen in cortical circuits. As a possible remedy, balanced amplification constitutes a putative mechanism for transient amplification in recurrent neural networks (Murphy and Miller, 2009). However, to achieve strong amplification, several ensembles need to be chained together into a hidden feedforward structure which manifests in strongly non-normal recurrent connectivity (Goldman, 2009; Hennequin et al., 2012, 2014; Bondanelli and Ostojic, 2020; Gillett et al., 2020). Yet, such network structures are at odds with the often observed symmetric excitatory connectivity (Ko et al., 2011; Cossell et al., 2015).

We are thus faced with a conundrum. On the one hand, Hebbian assemblies, whose connectivity is consistent with neurobiology, can amplify specific stimuli. But, the resulting persistent attractor dynamics are inconsistent with the transient activity observed in experiments. On the 
other hand, non-normal connectivity offers an appealing explanation for transient amplification in recurrent neural network models, but it is at odds with the mainly observed symmetric connectivity. Importantly, however, previous studies largely ignored STP, which considerably modulates synaptic transmission and shapes neural responses on timescales ranging from milliseconds to minutes (Tsodyks and Markram, 1997; Markram et al., 1998; Zucker and Regehr, 2002; Pala and Petersen, 2015). This raises the question of whether and how STP or other neuronal adaptation mechanisms could resolve the puzzle by reconciling the seemingly disparate aspects.

Here we address this question by studying the emergence of transient dynamics in recurrent network models and examine how they are shaped through neuronal nonlinearities, STP, and EI cotuning. Specifically, we first characterize the conditions under which individual neuronal ensembles with symmetric excitatory connectivity succumb to explosive run-away activity in response to external stimulation. We then show how STP can effectively mitigate this instability by re-stabilizing ensemble dynamics in an inhibition-stabilized network (ISN) state, but only after generating a pronounced stimulus-triggered onset transient. We call this mechanism nonlinear transient amplification (NTA) and show that it yields selective onset responses that carry more relevant stimulus information than the subsequent steady-state. Finally, we characterize the functional benefits of global El balance and co-tuning for NTA. We find that pattern classification in networks with NTA is enhanced by El balance in individual neurons, a feature that is widely observed in the brain (Wehr and Zador, 2003; Froemke et al., 2007; Okun and Lampl, 2008; Rupprecht and Friedrich, 2018) and readily emerges in computational models endowed with activity-dependent plasticity of inhibitory synapses (Vogels et al., 2011). Importantly, NTA purports that, following transient amplification, neuronal ensembles settle into a stable ISN state, consistent with previous work on stabilized supralinear networks (SSNs) (Ahmadian et al., 2013; Rubin et al., 2015; Hennequin et al., 2018; Kraynyukova and Tchumatchenko, 2018). In summary, our work indicates that NTA is ideally suited to amplify stimuli rapidly through the interaction of symmetric recurrent excitation with STP.

\section{Results}

To understand the emergence of transient responses in recurrent neural networks, we studied rate-based population models with a supralinear, power law input-output function (Fig. 1A, B; Ahmadian et al., 2013; Hennequin et al., 2018), which captures essential aspects of neuronal activation (Priebe et al., 2004), while also being analytically tractable. We first considered an isolated neuronal ensemble consisting of one excitatory (E) and one inhibitory (I) population (Fig. 1A).

The dynamics of this network are given by

$$
\tau_{E} \frac{d r_{E}}{d t}=-r_{E}+\left[J_{E E} r_{E}-J_{E l} r_{l}+g_{E}\right]_{+}^{\alpha_{E}}
$$




$$
\tau_{l} \frac{d r_{l}}{d t}=-r_{l}+\left[J_{I E} r_{E}-J_{l I} r_{l}+g_{l}\right]_{+}^{\alpha_{I}}
$$

where $r_{E}$ and $r_{l}$ are the firing rates of the excitatory and inhibitory population, $\tau_{E}$ and $\tau_{l}$ represent the corresponding time constants, $J_{X Y}$ denotes the synaptic strength from the population $Y$ to the population $X$, where $X, Y \in\{E, l\}, g_{E}$ and $g_{l}$ are the external inputs to the respective populations. Finally, $\alpha_{E}$ and $\alpha_{l}$, the exponents of the respective input-output functions, are fixed at two unless mentioned otherwise. For ease of notation, we further define the weight matrix $\mathbf{J}$ of the compound system as follows:

$$
\mathbf{J}=\left[\begin{array}{ll}
J_{E E} & -J_{E I} \\
J_{I E} & -J_{I I}
\end{array}\right]
$$

To account for the strong reciprocal E-to-E synaptic connections (Ko et al., 2011; Cossell et al., 2015), we studied networks in which the determinant

$$
\operatorname{det} \mathbf{J}=-J_{E E} J_{I I}+J_{I E} J_{E I}
$$

is negative. To mimic sensory stimulation, we investigated ensemble dynamics as a function of external input strength $g_{E}$. Importantly, we assumed that most inhibition originates from recurrent connections and (Franks et al., 2011; Large et al., 2016), hence, we kept the input to the inhibitory population $g_{l}$ fixed.

\section{Nonlinear amplification of inputs above a critical threshold}

We initialized the network in a stable low-activity state in the absence of external stimulation, consistent with spontaneous activity in cortical networks (Fig. 1C). However, an input $g_{E}$ of sufficient strength, destabilized the network (Fig. 1C). Importantly, this behavior is distinct from linear network models in which the network stability is independent of inputs (Methods). The transition from stable to unstable dynamics can be understood by examining the phase portrait of the system (Fig. 1D). Before stimulation, the system has a stable and an unstable fixed point (Fig. 1D, left). However, both fixed points disappear for an input $g_{E}$ above a critical stimulus strength (Fig. 1D, right).

To further understand the system's bifurcation structure, we consider the characteristic function

$$
F(z)=J_{E E}[z]_{+}^{\alpha_{E}}-J_{E I}\left[\operatorname{det} \mathbf{J} \cdot J_{E I}^{-1}[z]_{+}^{\alpha_{E}}+J_{E I}^{-1} J_{I I} z-J_{E I}^{-1} J_{I I} g_{E}+g_{l}\right]_{+}^{\alpha_{l}}-z+g_{E}
$$

where $z$ denotes the total current into the excitatory population and $\operatorname{det} \mathbf{J}$ represents the determinant of the weight matrix (Kraynyukova and Tchumatchenko, 2018; Methods). The characteristic function reduces the original two-dimensional system to one dimension, whereby the zero crossings of the characteristic function correspond to the fixed points of the original system (cf. Eq. 1-2). We use this correspondence to visualize how the fixed points of the system change with the input 
A
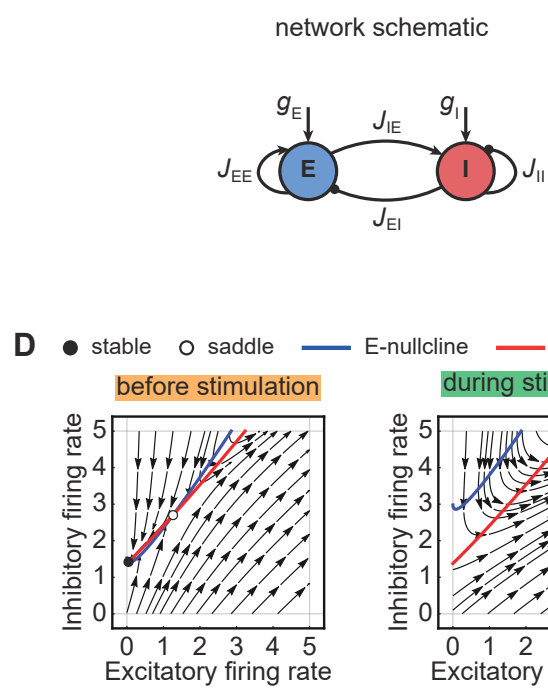

B

supralinear input-output function

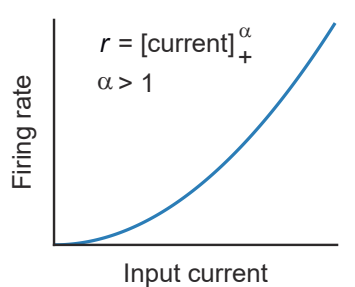

E

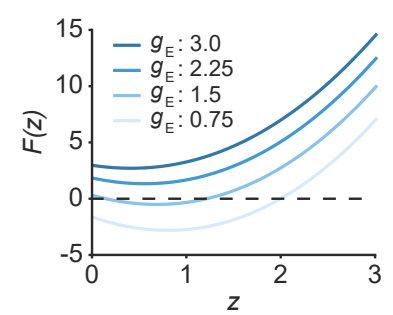

C

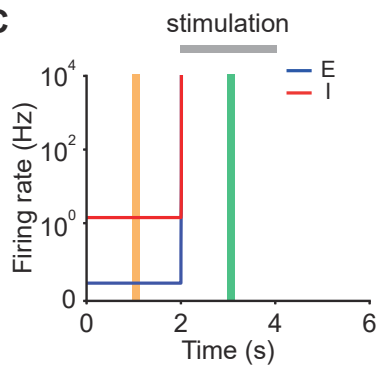

$\mathbf{F}$

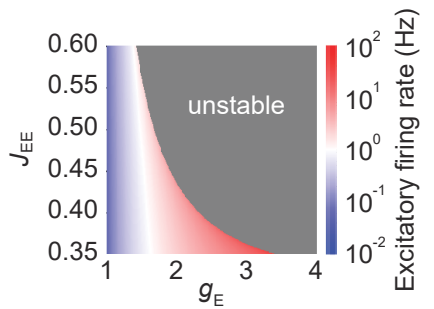

Fig. 1. Neuronal ensembles nonlinearly amplify inputs above a critical threshold. (A) Schematic of the recurrent ensemble model consisting of an excitatory (blue) and an inhibitory population (red). (B) Supralinear input-output function given by a rectified power law with exponent $\alpha=2$. (C) Firing rates of the excitatory (blue) and inhibitory population (red) in response to external stimulation during the interval from 2-4s (gray bar). The stimulation was implemented by temporarily increasing the input $g_{E}$. (D) Phase portrait of the system before stimulation (left; cf. C orange) and during stimulation (right; cf. $C$ green). (E) Characteristic function $F(z)$ for varying input strength $g_{E}$. Note that the function loses its zero crossings, which correspond to fixed points of the system for increasing external input. (F) Heat map showing the evoked firing rate of the excitatory population for different parameter combinations $J_{E E}$ and $g_{E}$. The gray region corresponds to the parameter regime with unstable dynamics.

$g_{E}$. Increasing $g_{E}$ shifts $F(z)$ upwards, which eventually leads to all zero crossings disappearing and the ensuing unstable dynamics (Fig. 1E; Methods). Importantly, for any weight matrix $\mathbf{J}$ with negative determinant, there exists a critical input $g_{E}$ at which all fixed points disappear (Methods). While for weak recurrent E-to-E connection strength $J_{E E}$, the transition from stable dynamics to unstable is gradual, in that it happens at higher firing rates (Fig. 1F), it becomes more abrupt for stronger $J_{E E}$. Thus, our analysis demonstrates that individual neuronal ensembles with negative determinant det $\mathbf{J}$ nonlinearly amplify inputs above a critical threshold by switching from initially stable to unstable dynamics.

\section{Short-term plasticity, but not spike-frequency adaptation, can re-stabilize ensemble dynamics}

Since unstable dynamics are not observed in neurobiology, we wondered whether neuronal spikefrequency adaptation (SFA) or STP could re-stabilize the ensemble dynamics while keeping the nonlinear amplification character of the system. Specifically, we considered SFA of excitatory neurons, E-to-E short-term depression (STD), and E-to-I short-term facilitation (STF). We focused on these particular mechanisms because they are ubiquitously observed in the brain. Most pyrami- 
dal cells exhibit SFA (Barkai and Hasselmo, 1994) and most synapses show some form of STP (Markram et al., 1998; Zucker and Regehr, 2002; Pala and Petersen, 2015). Moreover, the time scales of these mechanisms are well-matched to typical timescales of perception, i.e., from milliseconds to seconds (Tsodyks and Markram, 1997; Fairhall et al., 2001; Pozzorini et al., 2013).

When we simulated our model with weak SFA, it did not stabilize run-away excitation (Fig. 2A; Methods). Increasing the strength of SFA eventually led to oscillatory ensemble activity (Fig. S1). To understand this behavior, we analyzed the corresponding characteristic function $F(z)$ for an ensemble with SFA. We found that in the presence of SFA, $F(z)$ can maximally have one stable low-activity fixed point and one unstable high-activity fixed point (Fig. S1). This property is closely related to SFA's tendency to linearize a neuron's input-output function (Ermentrout, 1998; Benda and Herz, 2003) but not to saturate it. Thus, when an input causes the system's fixed points to disappear (cf. Fig. 1D, E), weak SFA either does not restore any fixed point, or the high-activity fixed point cannot "catch up" with the run-away dynamics. Therefore, the system's dynamics remain unstable. Strong SFA, on the other hand, allows the high-activity fixed point to catch up with the increasing current into the excitatory population $z$, but since it is an unstable fixed point, this results in a reduction of the excitatory ensemble activity toward the low-activity fixed point. Unfortunately, this change is only short-lived and as the adaptation variable recovers (cf. Eq. (21)), the ensemble activity engages in another cycle of explosive run-away activity. Thus, while the input is present, strong SFA creates a stable limit cycle with associated oscillatory ensemble activity (Fig. S1, Methods), which was also shown in previous modeling studies (Van Vreeswijk and Hansel, 2001), but is not typically observed in neurobiology (DeWeese et al., 2003; Mazor and Laurent, 2005; Rupprecht and Friedrich, 2018).

Next, we considered STP, which is capable of saturating the effective neuronal input-output function (Mongillo et al., 2012; Zenke et al., 2015). We first analyzed the stimulus-evoked network dynamics when we added STD to the recurrent E-to-E connections. Strong depression of synaptic efficacy resulted in a brief onset transient after which the ensemble dynamics quickly settled into a stimulus-evoked steady-state with slightly higher activity than the baseline (Fig. 2B, left). After stimulus removal, the ensemble activity returned back to its baseline level (Fig. 2B, left; Fig. 2C). Notably, the ensemble dynamics remained stable, albeit at a much higher firing rate, when inhibition was inactivated during stimulus presentation (Fig. 2B, right). This shows that STP is capable of creating a stable high-activity fixed point, which is fundamentally different from the SFA dynamics discussed above. This difference in ensemble dynamics can be readily understood by analyzing the self-consistent solutions of $F(z)$. Initially, the ensemble is at the stable low activity fixed point. But the stimulus causes this fixed point to disappear, thus giving way to positive feedback which creates the leading edge of the onset transient (cf. Fig. 2B). However, because E-to-E synaptic transmission is rapidly reduced by STD, the curvature of $F(z)$ changes and a stable fixed point is created, thereby allowing excitatory run-away dynamics to terminate and the ensemble 
dynamics settle into a steady-state at low activity levels (Fig. 2D). We found that E-to-I STF leads to similar dynamics (Fig. 2E, left) with the only difference that this configuration requires inhibition for network stability (Fig. 2E, right), whereas E-to-E STD stabilizes activity even without inhibition, albeit at physiologically implausibly high activity levels. Importantly, the re-stabilization through either form of STP did not impair an ensemble's ability to amplify stimuli during the initial onset phase.

To highlight the amplification power of supralinear networks over purely linear networks with equivalent weight strengths, we calculated the ratio of the evoked peak amplitudes of the firing rate to the input strength, henceforth called the "Amplification index". Notably, amplification of stimuli above the critical threshold in supralinear networks is orders of magnitude larger than in a linear network with comparable weights (Fig. $2 \mathrm{~F}$ ). We stress that the resulting high firing rates are parameter-dependent (Fig. S2), but also due to the short duration of the onset peak. In experiments, such high rates are observed as precisely time-locked spikes (DeWeese et al., 2003; Wehr and Zador, 2003; Bolding and Franks, 2018; Gjoni et al., 2018).

Furthermore, we investigated how the network operating regime changes with the stimulation. Recent studies suggested that cortical networks operate as inhibition-stabilized networks (ISNs) (Sanzeni et al., 2020), in which the excitatory network is unstable in the absence of feedback inhibition (Tsodyks et al., 1997). Whether a network is an ISN or not is mathematically determined by the real part of the leading eigenvalue of the Jacobian of the excitatory-to-excitatory subnetwork (Tsodyks et al., 1997). We computed the leading eigenvalue in our model and referred to it as "ISN index" in the following (Methods). We found that the ISN index switches sign from negative to positive during external stimulation, indicating that the ensemble transitions from a non-ISN to an ISN (Fig. 2G). Notably, this behavior is distinct from linear network models in which the network operating regime is independent of the input (Methods). One defining characteristic of ISNs is that injecting excitatory (inhibitory) current into inhibitory neurons decreases (increases) inhibitory firing rates, which is also known as the paradoxical effect (Tsodyks et al., 1997; Miller and Palmigiano, 2020). To illustrate the difference in network operating regimes in terms of the paradoxical effect, we injected excitatory current into the inhibitory population before and during stimulus presentation. We found that before stimulation, the network did not exhibit the paradoxical effect (Fig. 2H, left; Fig. S3). In contrast, injecting excitatory inputs into the inhibitory population during stimulation reduced their activity (Fig. $2 \mathrm{H}$, right; Fig. S3). Thus, during stimulation the neuronal ensemble switches to an ISN state.

Despite the fact that the supralinear input-output function of our framework captures some aspects of intracellular recordings (Priebe et al., 2004), it is unbounded and thus allows infinitely high firing rates. This is in contrast to neurobiology where firing rates are bounded due to neuronal refractory effects. While this assumption permitted us to analytically study the system and therefore to gain a deeper understanding of the underlying ensemble dynamics, we wondered whether our main 
bioRxiv preprint doi: https://doi.org/10.1101/2021.06.09.447718; this version posted June 10,2021. The copyright holder for this preprint (which was not certified by peer review) is the author/funder, who has granted bioRxiv a license to display the preprint in perpetuity. It is made available under aCC-BY 4.0 International license.

A

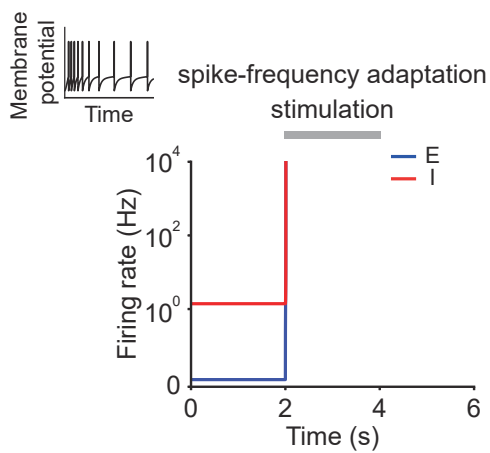

C

- fixed point before/after stim.

- fixed point during stim.

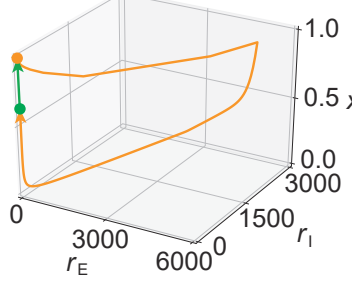

$\mathbf{F}$

E-to-E STD

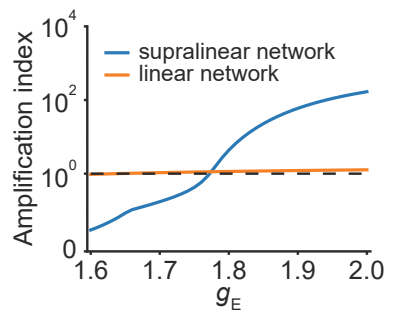

E-to-E STD

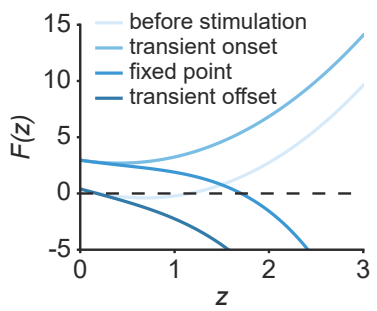

G

E-to-I STF

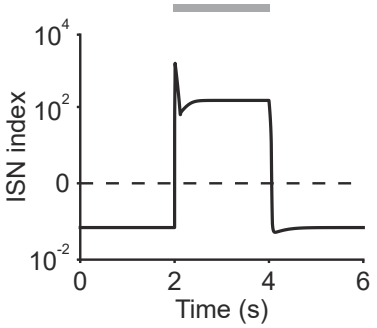

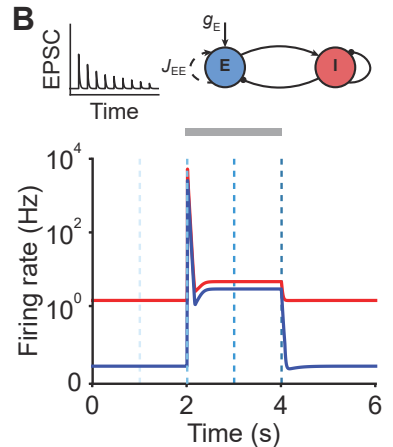

E-to-E STactivate

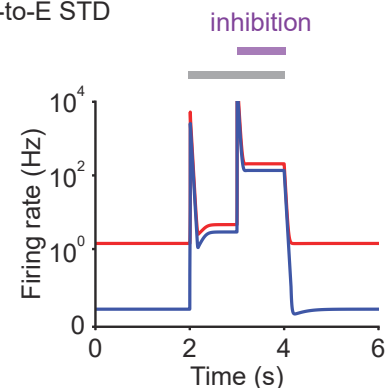

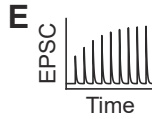

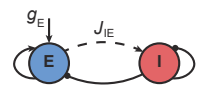

E-to-I STF inactivate
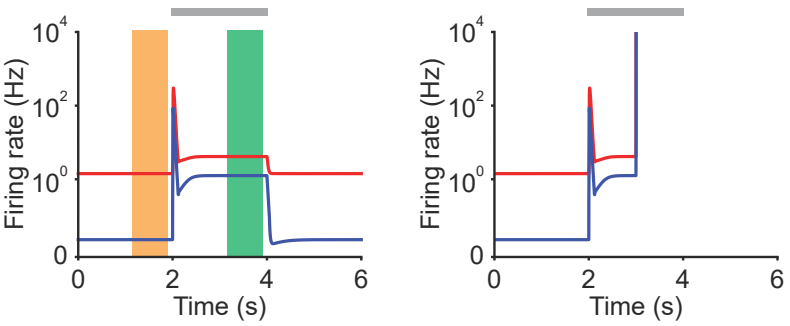

H

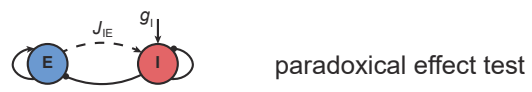

before stimulation
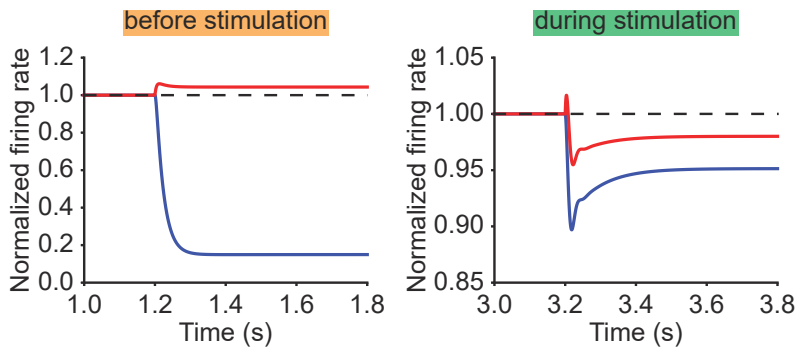

Fig. 2. Short-term plasticity, but not spike-frequency adaptation, re-stabilizes ensemble dynamics. (A) Firing rates of the excitatory (blue) and inhibitory population (red) in the presence of spike-frequency adaptation (SFA). During stimulation (gray bar) additional input is injected into the excitatory population. The inset shows a cartoon of how SFA affects spiking neuronal dynamics in response to a step current input. (B) Left: Same as (A) but in the presence of Eto-E short-term depression (STD). Right: Same as left but inactivating inhibition in the period marked in purple. (C) 3D plot of the excitatory activity $r_{E}$, inhibitory activity $r_{l}$, and the STD variable $x$ of the network in B left. The orange and green points mark the fixed points before/after and during stimulation. (D) Characteristic function $F(z)$ in networks with E-to-E STD. Different brightness levels correspond to different time points in B left. (E) Same as (B) but in the presence of E-to-I short-term facilitation (STF). (F) Amplification index, which is defined as the ratio of the peak amplitude to input $g_{E}$, as a function of input $g_{E}$ for supralinear networks (blue) and linear networks (orange) in the presence of E-to-E STD. (G) inhibition-stabilized network (ISN) index, which corresponds to the largest real part of the eigenvalues of the Jacobian matrix of the E-E subnetwork, as a function of time for the network with E-to-I STF in E left. For values above zero (dashed line), the ensemble is an ISN. (H) The normalized firing rates of the excitatory (blue) and inhibitory population (red) when injecting additional excitatory current into the inhibitory population before stimulation (left; cf. orange bar in E), and during stimulation (right; cf. green bar in E). Initially, the ensemble is in the non-ISN regime and injecting excitatory current into the inhibitory population increases its firing rate. During stimulation, however, the ensemble is an ISN. In this case, excitatory current injection into the inhibitory population results in a reduction of its firing rate, also known as the paradoxical effect. 
conclusions were also valid when we limited the maximum firing rates. To that end, we carried out the same simulations while capping the firing rate at $300 \mathrm{~Hz}$. In the absence of additional SFA or STP mechanisms, the firing rate saturation introduced a stable high-activity state in the ensemble dynamics which replaced the unstable dynamics in the uncapped model. As above, the ensemble entered this high-activity steady-state when stimulated with an external input above a critical threshold and exhibited persistent activity after stimulus removal (Fig. S4). While weak SFA did not change this behavior, strong SFA resulted in oscillatory behavior during stimulation consistent with previous analytical work (Fig. S4, Van Vreeswijk and Hansel, 2001), but did not in stable steady-states commonly observed in biological circuits. In the presence of E-to-E STD or E-to-I STF, however, the ensemble exhibited transient evoked activity at stimulation onset that was comparable to the uncapped case. Importantly, the ensemble did not show persistent activity after the stimulation (Fig. S4). Finally, we confirmed that all of these findings were qualitatively similar in a realistic spiking neural network model (Fig. S5; Methods).

In summary, we found that neuronal ensembles can rapidly, nonlinearly, and transiently amplify inputs by briefly switching from stable to unstable dynamics before being re-stabilized through STP mechanisms. We call this mechanism nonlinear transient amplification (NTA) which, in contrast to balanced amplification (Murphy and Miller, 2009; Hennequin et al., 2012), arises from nonlinear population dynamics interacting with STP. NTA is characterized by a large onset response, a subsequent ISN steady-state while the stimulus persists, and a return to a unique baseline activity state after the stimulus is removed. Thus, NTA is ideally suited to rapidly and nonlinearly amplify sensory inputs through symmetric recurrent excitatory connections, like reported experimentally (Ko et al., 2011; Cossell et al., 2015), while avoiding persistent activity.

\section{Co-tuned inhibition broadens the parameter regime of NTA}

Up to now, we have focused on a single neuronal ensemble. However, to process information in the brain, several ensembles with different stimulus selectivity presumably coexist and interact in the same circuit. This coexistence creates potential problems. It can lead to multi-stable persistent attractor dynamics, which are not commonly observed and could have adverse effects on the processing of subsequent stimuli. One solution to this issue could be El co-tuning, which arises in network models with plastic inhibitory synapses (Vogels et al., 2011) and has been observed experimentally in several sensory systems (Wehr and Zador, 2003; Froemke et al., 2007; Okun and Lampl, 2008; Rupprecht and Friedrich, 2018).

To characterize the conditions under which neuronal ensembles nonlinearly amplify stimuli without persistent activity, we analyzed the case of two interacting ensembles. More specifically, we considered networks with two excitatory ensembles and distinguished between global and co-tuned inhibition (Fig. 3A). In the case of global inhibition, one inhibitory population non-specifically inhibits both excitatory populations (Fig. 3A, left). In contrast, in networks with co-tuned inhibition, 
A

global inhibition

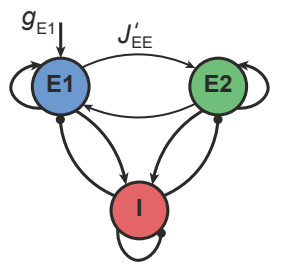

B $\Delta$ bi/multi-stable stimulation

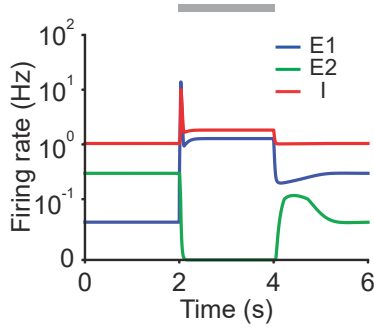

C

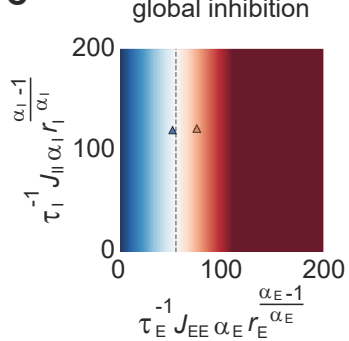

co-tuned inhibition

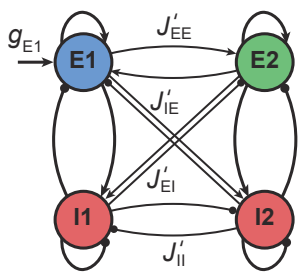

$\Delta$ uni-stable

stimulation

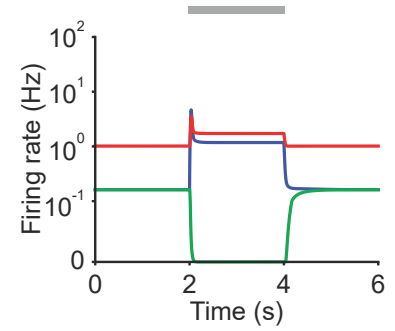

co-tuned inhibition

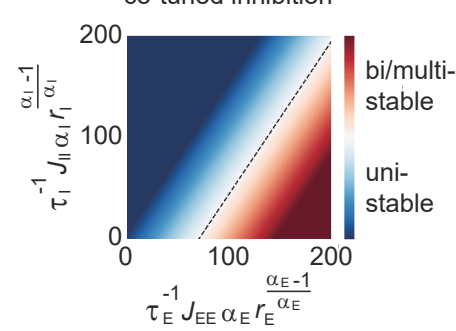

Fig. 3. Co-tuned inhibition broadens the parameter regime of NTA.

(A) Schematic of two neuronal ensembles with global inhibition (left) and with co-tuned inhibition (right). (B) Firing rate dynamics of bi/multi-stable ensemble dynamics (left) and uni-stable (right). In both cases, additional excitatory inputs are injected into excitatory ensemble $E 1$ during the period marked in gray. (C) Analytical solution of uni- and bi/multi-stability regions for global inhibition (left) and co-tuned inhibition (right). Co-tuning results in a larger parameter regime of uni-stability. The triangles correspond to the two examples in $\mathrm{B}$.

each ensemble is formed by a dedicated pair of an excitatory and an inhibitory population which can have cross-over connections, for instance, due to overlapping ensembles (Fig. 3A, right).

Global inhibition supports winner-take-all competition and is therefore often associated with multistable attractor dynamics (Wong and Wang, 2006; Mongillo et al., 2008). We first illustrated this effect in a network model with global inhibition. When the recurrent excitatory connections within each ensemble were sufficiently strong, small amounts of noise in the initial condition led to one of the ensembles spontaneously activating at elevated firing rates, while the other ensemble's activity remained low (Fig. 3B, left). A specific external stimulation could trigger a switch from one state to the other in which the other ensemble was active at a high firing rate. Importantly, this change persisted even after the stimulus had been removed, a hallmark of multi-stable dynamics. In contrast, uni-stable systems have a global symmetric state in which both ensembles have the same activity in the absence of stimulation. While the stimulated ensemble showed elevated firing rates in response to the stimulus, its activity returned to the baseline level after the stimulus is removed (Fig. 3B, right), consistent with experimental observations (DeWeese et al., 2003; Rupprecht and Friedrich, 2018; Bolding and Franks, 2018). Note that the only difference between these two models is that $J_{E E}$ is larger in the multi-stable example than in the uni-stable one.

Symmetric baseline activity is most consistent with activity observed in sensory areas. Hence, 
we sought to understand which inhibitory connectivity would be most conducive to maintain it. To that end, we analytically identified the uni-stability conditions, which are determined by the leading eigenvalue of the Jacobian matrix of the system, for networks with varying degrees of El co-tuning (Methods). We found that a broader parameter regime underlies uni-stability in networks with cotuned inhibition than global inhibition (Fig. 3C). Notably, this conclusion is general and extends to networks with an arbitrary number of ensembles (Methods). However, co-tuning does not impair an ensemble's ability to exhibit NTA as shown above for an isolated ensemble with perfect cotuning. Thus, co-tuned inhibition helps to avoid persistent attractor dynamics and broadens the parameter regime of uni-stability without adversely affecting NTA.

\section{NTA provides better pattern completion and pattern separation than fixed points}

Neural circuits are capable of generating stereotypical activity patterns in response to partial cues and forming distinct representations in response to different stimuli. To test whether NTA achieves pattern completion and supports pattern separation, we analyzed the transient onset activity in our models and compared it to the fixed point activity.

To investigate pattern completion and pattern separation in our model, we considered a co-tuned network with E-to-E STD and two distinct excitatory ensembles $E 1$ and $E 2$. We gave additional input $g_{E 1}$ to a Subset 1 , consisting of $75 \%$ of the neurons in ensemble $E 1$ (Fig. 4A). We then measured the evoked activity in the remaining $25 \%$ of the excitatory neurons in $E 1$ to quantify pattern completion. To assess pattern separation, we injected additional input $g_{E 1}$ into the $E 1$ neurons during the second stimulation phase (Fig. 4A) while measuring the activity of E2. Interestingly, we found that neurons in Subset 2, which did not receive additional input, showed large onset responses, their steady-state activity was largely suppressed (Fig. 4B). Despite the fact that inputs to $E 1$ caused increased transient onset responses in $E 2$, the amount of increase was orders of magnitude smaller than in $E 1$ (Fig. 4B). To quantify pattern completion, we defined the

$$
\text { Association Index }=1+\frac{r_{E 1_{2}}-r_{E 1_{1}}}{r_{E 1_{2}}+r_{E 1_{1}}}
$$

Here, $r_{E 1_{1}}$ and $r_{E 1_{2}}$ correspond to the subpopulation activities of $E 1$, respectively. As per our definition, the Association Index ranges from zero to one, with larger values indicating stronger associativity. In addition, to quantify the separation between $E 1$ and $E 2$, we considered a binary classifier tasked to distinguish the two input stimuli and measured the distance to the classifier's decision boundary, whereby larger values indicate a larger classification margin and thus better separability (Methods). Note that the Association Index and the distance to the decision boundary were computed from different input configurations corresponding to different phases in our simulation paradigm (Fig. 4B).

With these definitions, we ran simulations with different input strengths $g_{E 1}$. We found that the onset peaks showed stronger association than the fixed-point activity (Fig. 4C). Note that the 
A pattern completion $(75 \%$ of $\mathrm{E} 1)$ pattern separation

phase 2: activate E1 subset 1 and 2

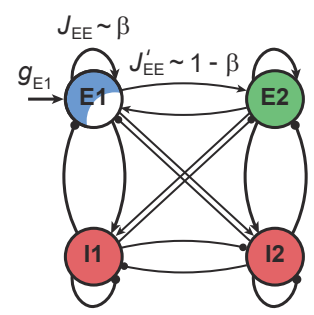

C

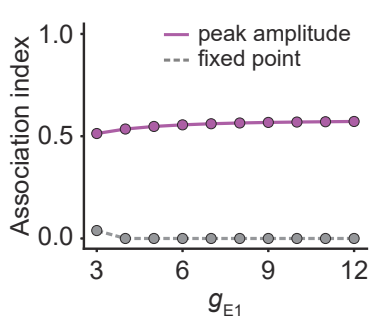

D

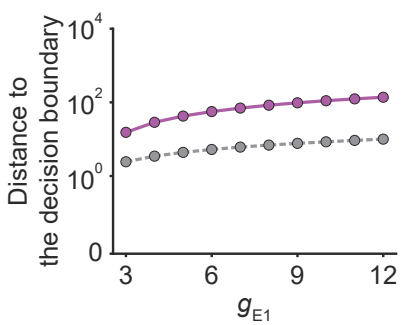

B

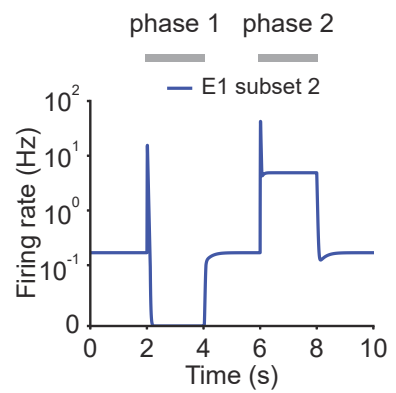

E

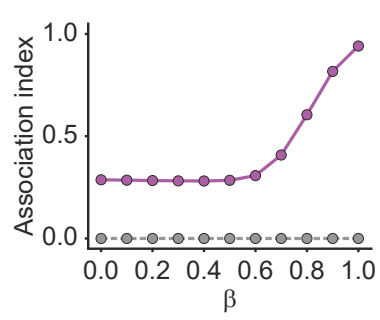

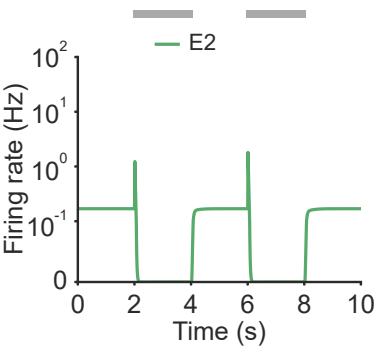

$\mathbf{F}$

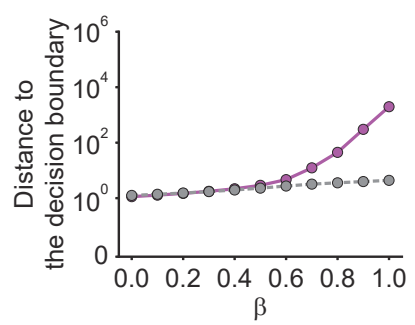

Fig. 4. NTA yields stronger pattern completion and pattern separation than fixed points. (A) Schematic of the network setup used to probe pattern completion and pattern separation. To assess the effect on pattern completion, $75 \%$ of the neurons (Subset 1 ) in ensemble $E 1$ received additional input $g_{E 1}$ during Phase 1 (2-4 s), while we recorded the firing rate of the remaining $25 \%$ (Subset 2 ) in the excitatory ensemble $E 1$. To evaluate the impact on pattern separation, all neurons in $E 1$ received additional inputs $g_{E 1}$ in Phase 2 (6-8s) while the firing rate of $E 2$ was measured. (B) Examples of firing rates of Subset 2 of $E 1$ (left, blue) and $E 2$ (right, green) with E-to-E STD. (C) Association index as a function of input $g_{E 1}$ for the onset peak amplitude (magenta solid line) and fixed-point activity (gray dashed line) for E-to-E STD. (D) Distance to the decision boundary as a function of input $g_{E 1}$ for the onset peak amplitude (magenta solid line) and fixed-point activity (gray dashed line) for E-to-E STD. (E and F) Same as C and D but as a function of $\beta$, which controls the inner- and inter-ensemble connection strength.

association index at the fixed point remained zero, a direct consequence of $r_{E 1_{2}}$ being suppressed to zero (Fig. 4C). Furthermore, we found that the separation between the transient onset response and the decision boundary was always greater than for the fixed-point activity (Fig. 4D) showing that onset responses provide better pattern separation than fixed points.

To investigate how the recurrent excitatory connectivity affects both pattern completion and pattern separation, we introduced the parameter $\beta$ which controls the within-ensemble E-to-E strength $J_{E E}$ relative to the inter-ensemble strength $J_{E E}^{\prime}$ (Fig. $4 \mathrm{~A}$ ) such that $J_{E E}=\beta J_{\text {tot }}$ and $J_{E E}^{\prime}=(1-\beta) J_{\text {tot }}$. These definitions ensure that the total weight $J_{t o t}=J_{E E}+J_{E E}^{\prime}$ remains constant for any choice of $\beta$. Notably, the overall recurrent excitation strength within an ensemble $J_{E E}$ increases with increasing $\beta$. When $\beta$ is larger than 0.5 , the excitatory connection strength within the ensemble $J_{E E}$ exceeds the one between ensembles $J_{E E}^{\prime}$.

We found that NTA's pattern completion and separation capabilities monotonically increase with $\beta$ (Fig. 4E, F), confirming that recurrent excitatory strength is a key determinant of network dynamics. Finally, we confirmed that our findings were also valid in networks with E-to-I STF (Fig. S6), which 
A

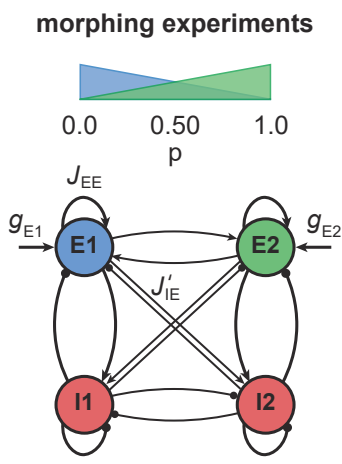

B

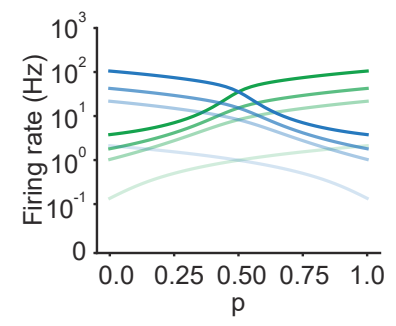

D $\quad-$ peak --- fixed point

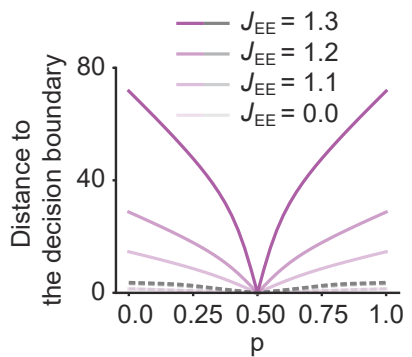

C
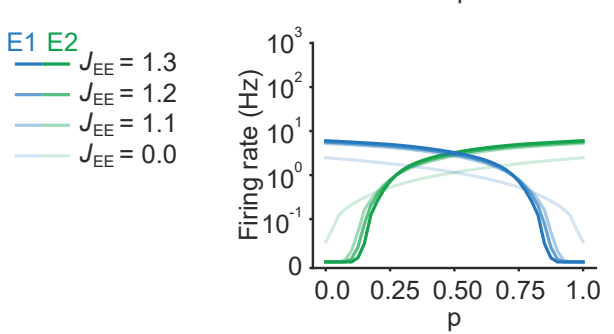

E

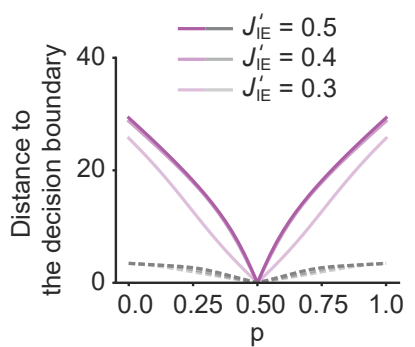

Fig. 5. NTA provides stronger amplification and pattern separation in morphing experiments than fixed point activity. (A) Schematic of the morphing stimulation paradigm. The fraction of the additional inputs into the two excitatory ensembles is controlled by the parameter $p$. (B) Peak amplitude of $E 1$ (blue) and E2 (green) as a function of $p$. Brightness levels represent different recurrent E-to-E connection strengths $J_{E E}$. (C) Same as in B but for fixed-point activity. (D) Distance to the decision boundary as a function of $p$ for the peak onset response (magenta solid line) and fixed-point activity (gray dashed line). (E) Same as D but with different E-to-I connection strengths $J_{I E}^{\prime}$ across ensembles.

is commonly observed in the brain (Markram et al., 1998; Zucker and Regehr, 2002; Pala and Petersen, 2015). In summary, NTA's transient onset responses result in overall better pattern completion and pattern separation than fixed point activity.

\section{NTA provides higher amplification and pattern separation in morphing experiments}

So far, we only considered input to one ensemble. To examine how representations in our model are affected by ambiguous inputs to several ensembles, we performed additional morphing experiments (Freedman et al., 2001; Niessing and Friedrich, 2010). To that end, we introduced the parameter $p$ which interpolates between two input stimuli which target $E 1$ and $E 2$ respectively. When $p$ is zero, all additional input is injected into $E 1$. For $p$ equal to one, all additional input is injected into $E 2$. Finally, $p$ equal to 0.5 corresponds to the symmetric case in which $E 1$ and $E 2$ receive the same amount of additional input (Fig. 5A).

First, we investigated how the recurrent excitatory connection strength within each ensemble $J_{E E}$ affects the onset peak amplitude and fixed-point activity. We found that the peak amplitudes depend strongly on $J_{E E}$, whereas the fixed-point activity was only weakly dependent on $J_{E E}$ (Fig. 5B, C). When we disconnected the ensembles by completely eliminating all recurrent excitatory con- 
nections, activity was noticeably decreased (Fig. 5B, C). This illustrates, that recurrent excitation does play an important role in selectively amplifying specific stimuli similar to experimental observations (Marshel et al., 2019; Peron et al., 2020), but that amplification is highest at the onset.

Further, we examined the impact of competition through lateral inhibition as a function of the E-

to-I inter-ensemble strength $J_{I E}^{\prime}$ (Methods). As above, we quantified its impact by measuring the representational distance to the decision boundary for the transient onset responses and fixedpoint activity. We found that regardless of the specific STP mechanism, the distance was larger for the onset responses than for the fixed-point activity, consistent with the notion that the onset can encode the stimulus identity more reliably than the fixed-point (Fig. 5D-E, Fig. S7). Thus, NTA provides stronger amplification and pattern separation than fixed points in response to ambiguous stimuli.

\section{Nonlinear transient amplification in spiking neural networks}

Thus far, our analysis relied on power law neuronal input-output functions in the interest of analytical tractability. To test whether our findings also qualitatively apply to more realistic network models, we built a spiking neural network consisting of randomly connected 800 excitatory and 200 inhibitory neurons, in which the E-to-E synaptic connections were subject to STD (Methods). Here, we defined five overlapping ensembles, each corresponding to 200 randomly selected excitatory neurons. During an initial simulation phase (0-22s), we consecutively stimulated each pattern by giving additional input to their excitatory neurons, whereas the input to other neurons remained unchanged (Fig. 6A). In addition, we also tested pattern completion by stimulating only $75 \%$ (Subset 1) of the neurons belonging to Pattern 5 (22-24 s; Fig. 6A). We quantified each pattern's activity by calculating the population firing rate of the stored patterns (Methods). As in the case of the rate-based model, the neuronal ensembles in the spiking model generated pronounced transient onset responses. We then measured the difference of peak pattern activity and steadystate activity between the stimulated pattern and the remaining unstimulated patterns (Methods). As for the rate-based networks, this difference was consistently larger for the onset peak than for the fixed-point (Fig. 6B, C). Thus, transient onset responses allow better stimulus separation than fixed points also in spiking neural network models.

Finally, to visualize the neural activity, we projected the binned spiking activity during the first $10 \mathrm{~s}$ of our simulation onto its first two principal components. Notably, the PC trajectory does not exhibit a pronounced rotational component (Fig. 6D) as activity is confined to one specific ensemble, consistent with experiments (Marshel et al., 2019). Furthermore, we computed the fifth pattern's activity for Subset 1 and 2 during the time interval 16-26s. In agreement with our rate models, neurons in Subset 2 which did not receive additional inputs showed a strong response at the onset (Fig. 6E), but not at the fixed point, suggesting that the strongest pattern completion occurs during the initial amplification phase. Thus, the key characteristics of NTA are preserved 
A
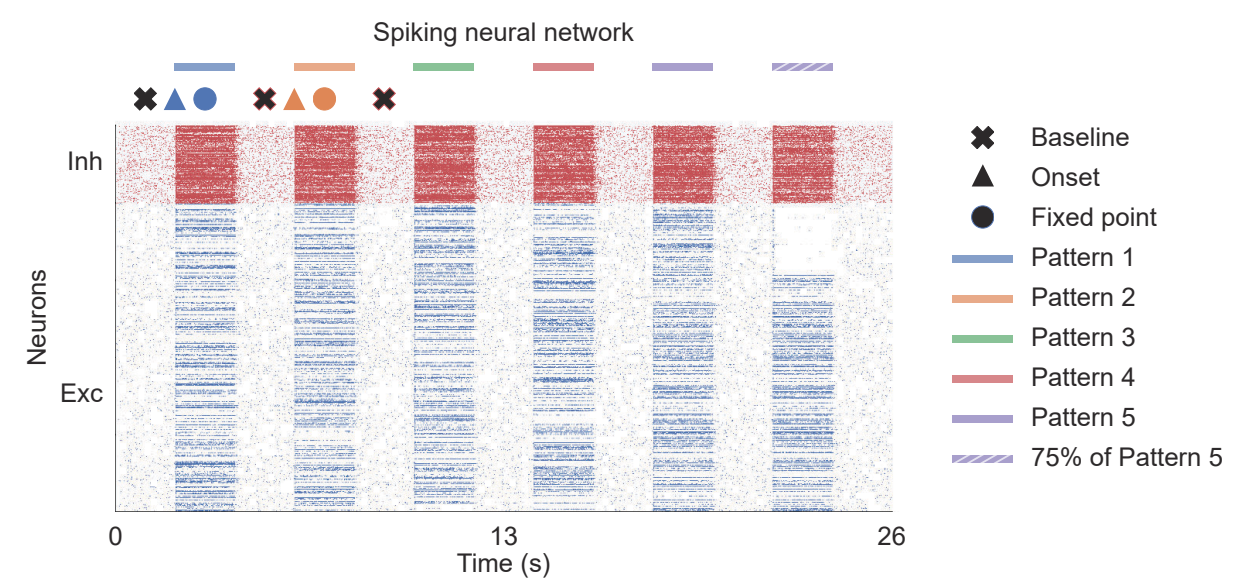
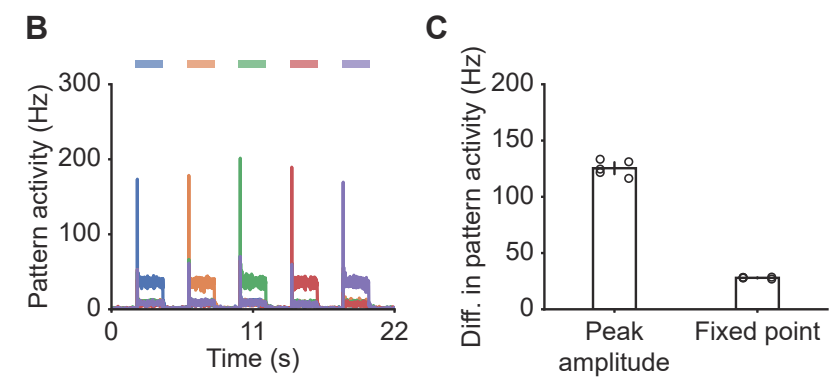

D
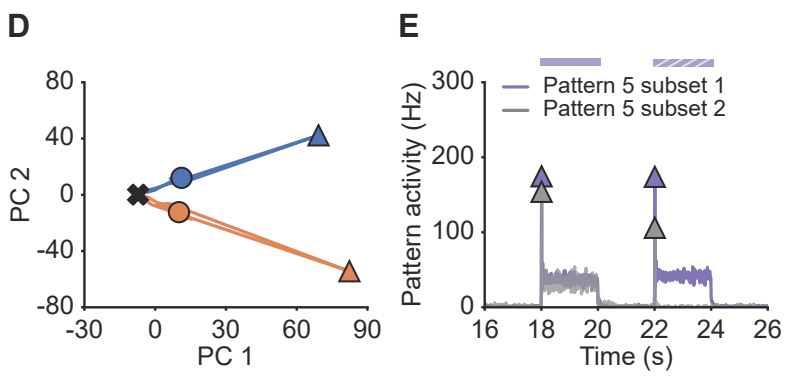

Fig. 6. Spiking neural network simulations qualitatively reproduce NTA dynamics of rate models. (A) Spiking activity of excitatory (blue) and inhibitory (red) neurons in a spiking neural network. From 2-20 s, Patterns 1-5 individually received additional input for $2 \mathrm{~s}$ each (colored bars). From $22-24 \mathrm{~s}, 75 \%$ of Pattern 5 neurons (Subset 1) received additional input, whereas the rest $25 \%$ of Pattern 5 neurons (Subset 2) did not receive additional input. The symbols at the top designate the different simulation phases of baseline activity, the onset transients, and the fixed point activity. Different colors correspond to the distinct stimulation periods. (B) Pattern activity of each stored pattern (colors). (C) Difference in pattern activity between the stimulated pattern with the remaining patterns for the transient onset peak and the fixed point. Points correspond to the different stimulation periods. (D) Spiking activity during the interval 0-10 s represented in the PCA basis spanned by the first two principal components which captured approximately $40 \%$ of the total variance. The colored lines represent the PC trajectories of the first two stimuli shown in A and B. Triangles, points and crosses correspond to the onset peak, fixed point, and baseline activity, respectively. (E) Pattern activity of Subset 1 (purple) and Subset 2 (gray) of Pattern five from 16-26s. Onset peaks are marked by triangles.

across rate-based and more realistic spiking neural network models.

\section{Discussion}

In this study, we demonstrated that neuronal ensemble models with recurrent excitation and suitable forms of STP exhibit nonlinear transient amplification (NTA), a putative mechanism underlying selective amplification in recurrent circuits. NTA combines a supralinear neuronal transfer function, recurrent excitation between neurons with similar tuning, and pronounced STP. Using analytical and numerical methods, we showed that NTA generates rapid transient onset responses during which optimal stimulus separation occurs rather than at steady-states. Additionally, we 
showed that co-tuned inhibition is conducive to prevent the emergence of persistent activity, which could otherwise interfere with processing subsequent stimuli. In contrast to balanced amplification (Murphy and Miller, 2009), NTA is an intrinsically nonlinear mechanism for which only stimuli above a critical threshold are amplified effectively. While the precise threshold value is parameterdependent, it can be arbitrarily low provided the excitatory recurrent connections are sufficiently strong (cf. Fig. 1F). Importantly, such a critical activation threshold offers a possible explanation for sensory perception experiments which show similar threshold behavior (Marshel et al., 2019; Peron et al., 2020). Following transient amplification, ensemble dynamics are inhibition-stabilized, which renders our model compatible with existing work on SSNs (Ahmadian et al., 2013; Rubin et al., 2015; Hennequin et al., 2018; Kraynyukova and Tchumatchenko, 2018). Thus, NTA provides a parsimonious explanation for why sensory systems may rely upon neuronal ensembles with recurrent excitation in combination with El co-tuning, and pronounced STP dynamics.

Several theoretical studies approached the problem of transient amplification in recurrent neural network models. One particularly well-studied mechanism relies on non-normal connectivity matrices whereby stimuli are selectively amplified through asymmetric synaptic connections with an implicit feedforward structure (Murphy and Miller, 2009; Goldman, 2009; Hennequin et al., 2014; Bondanelli and Ostojic, 2020; Gillett et al., 2020; Christodoulou et al., 2021). Importantly, nonnormal amplification can generate rich transient activity in linear network models but lacks a critical threshold above which amplification occurs. These properties contrast with NTA, which relies on a nonlinear transfer function and symmetric excitatory connections within neuronal ensembles with similar tuning. The symmetric connectivity generates potent run-away activity above a critical stimulus strength. Nevertheless, the overall network dynamics are stable because run-away dynamics are eventually quenched through STP. Crucially, after the transient amplification phase, ensemble dynamics settle in an inhibitory-stabilized state, which renders NTA compatible with previous work on SSNs only that in our case stabilization is accomplished dynamically through STP. Due to the switch of the network's dynamical state, NTA's amplification is orders of magnitudes larger than balanced amplification (Murphy and Miller 2009; cf. Fig. 2D, Fig. S8).

NTA requires STP and recurrent inhibition, whose role on network dynamics have been studied in the past. Yet, STP was mainly examined in the context of stable working memory in recurrent networks (Mongillo et al., 2008; Zenke et al., 2015; Seeholzer et al., 2019) and transient delay activity following stimulus offset (Hempel et al., 2000; Gillary et al., 2017). However, its role in generating strongly amplified onset transients as a possible coding paradigm was largely ignored. Recurrent inhibition is essential for NTA to ensure uni-stability and selectivity through the suppression of ensembles with different tuning. This requirement is similar in flavor to semi-balanced networks characterized by excess inhibition to some excitatory ensembles while others are balanced (Baker et al., 2020). However, the theory of semi-balanced networks has, so far, only been applied to steady-state dynamics while ignoring transients and STP. Previous work showed that 
STP can tune networks to a critical state (Levina et al., 2007), but focused primarily on E-to-E STD. Our work extends this notion by combining it with a distinct computational mechanism and shows that both E-to-E STD and E-to-I STF can re-stabilize ensemble dynamics. El co-tuning prominently features in several models and was shown to support network stability (Vogels et al., 2011; Hennequin et al., 2017; Znamenskiy et al., 2018), efficient coding (Denève and Machens, 2016), novelty detection (Schulz et al., 2020), changes in neuronal variability (Hennequin et al., 2018; Rost et al., 2018), and correlation structure (Wu et al., 2020). Moreover, some studies have argued that El balance and co-tuning could increase robustness to noise in the brain (Rubin et al., 2017). The present work mainly highlights its importance for preventing multi-stability and delay activity in circuits not requiring such long-timescale dynamics.

NTA is consistent with several experimental findings. First, our model recapitulates the key findings of Shew et al. (2015) who showed ex vivo that strong sensory inputs cause a transient shift to a supercritical state, after which adaptive changes rapidly tune the network to criticality. Second, NTA requires symmetric excitatory connectivity between neurons with similar tuning, which has been reported in experiments (Ko et al., 2011; Cossell et al., 2015; Peron et al., 2020). Third, ensemble activation in our model depends on a critical stimulus strength in line with recent alloptical experiments in the visual cortex, which further link ensemble activation with a perceptual threshold (Marshel et al., 2019). Fourth, sensory networks are uni-stable in that they return to a non-selective activity state after the removal of the stimulus and usually do not show persistent activity (DeWeese et al., 2003; Mazor and Laurent, 2005; Rupprecht and Friedrich, 2018). Fifth, our work shows that NTA's onset responses encode stimulus identity better than the fixed-point activity, consistent with experiments in the locust antennal lobe (Mazor and Laurent, 2005) and research supporting that the brain relies on coactivity on short timescales to represent information (Stopfer et al., 1997; Engel et al., 2001; Harris et al., 2003; El-Gaby et al., 2021). Finally, El cotuning, which is conducive for NTA, has been found ubiquitously in different sensory circuits (Wehr and Zador, 2003; Froemke et al., 2007; Okun and Lampl, 2008; Rupprecht and Friedrich, 2018; Znamenskiy et al., 2018).

In our model, we made several simplifying assumptions. For instance, we kept the input to inhibitory neurons fixed and only varied the input to the excitatory population. This step was motivated by experiments in the piriform cortex where the total inhibition is dominated by feedback inhibition (Franks et al., 2011). Nevertheless, significant feedforward inhibition was observed in other areas (Bissieére et al., 2003; Cruikshank et al., 2007; Ji et al., 2016; Miska et al., 2018). While an in-depth comparison for different origins of inhibition was beyond the scope of the present study, we found that increasing the inputs to the excitatory population and inhibitory population by the same amount can still lead to NTA (Fig. S9; Methods). Therefore, we are confident that our main findings remain unaffected in the presence of substantial feedforward inhibition. Similarly, we limited our analysis to only a few overlapping ensembles. It will be interesting future work to study 
NTA in the case of many interacting and potentially overlapping ensembles and to determine the maximum storage capacity above which performance degrades. Finally, we anticipate that temporal differences in excitatory and inhibitory synaptic transmission may be important to preserve NTA's stimuli selectivity.

Our model makes several predictions. In contrast to balanced amplification, in which the network operating regime depends solely on the connectivity, an ensemble involved in NTA transitions from a non-ISN to an ISN state. This transition is consistent with noise variability observed in sensory cortices (Hennequin et al., 2018) and could be tested experimentally by probing the paradoxical effect under different stimulation conditions (Fig. 2G-H, Fig. S3). Moreover, NTA predicts that onset activity provides a better stimulus encoding and its activity is correlated with the fixed-point activity. This signature is different from purely non-normal amplification mechanisms which would involve a wave of neuronal activity across several distinct ensembles similar to a synfire chain. The difference should be clearly discernible in data. Since NTA relies on symmetric excitation between ensemble neurons, it suggests normal dynamics in which distinct ensembles first activate and then inactivate. The resulting dynamics have weak rotational components (cf. Fig. 6D) as seen in some experiments (Marshel et al., 2019). Strong non-normal amplification, on the other hand, relies on sequential activation of multiple ensembles, associated with pronounced rotational dynamics (Hennequin et al., 2014; Gillett et al., 2020), as for instance observed in motor areas (Churchland et al., 2012). Although both non-normal mechanisms and NTA are likely to co-exist in the brain, we speculate that strong NTA is best suited for, and thus most like to be found in, sensory systems.

In summary, we introduced a general theoretical framework of selective transient signal amplification in recurrent networks. Our approach derives from the minimal assumptions of a nonlinear neuronal transfer function, symmetric excitation within neuronal ensembles, and STP. Importantly, our analysis revealed the functional benefits of STP and El co-tuning, both pervasively found in sensory circuits. Finally, our work suggests that transient onset responses rather than steady-state activity are ideally suited for coactivity-based stimulus encoding and provides several testable predictions. 


\section{Methods}

\section{Stability conditions for supralinear networks}

The dynamics of a neuronal ensemble consisting of one excitatory and one inhibitory population with a supralinear, power law input-output function can be described as follows:

$$
\begin{aligned}
\tau_{E} \frac{d r_{E}}{d t} & =-r_{E}+\left[J_{E E} r_{E}-J_{E l} r_{l}+g_{E}\right]_{+}^{\alpha_{E}} \\
\tau_{l} \frac{d r_{l}}{d t} & =-r_{l}+\left[J_{l E} r_{E}-J_{I I} r_{l}+g_{l}\right]_{+}^{\alpha_{l}}
\end{aligned}
$$

The Jacobian $\mathbf{M}$ of the system is given by

$$
\mathbf{M}=\left[\begin{array}{cc}
\tau_{E}^{-1}\left(J_{E E} \alpha_{E} r_{E}^{\frac{\alpha_{E}-1}{\alpha_{E}}}-1\right) & -\tau_{E}^{-1} J_{E l} \alpha_{E} r_{E}^{\frac{\alpha_{E}-1}{\alpha_{E}}} \\
\tau_{l}^{-1} J_{I E} \alpha_{l} r_{l}{ }^{\frac{\alpha_{l}-1}{\alpha_{l}}} & -\tau_{l}^{-1}\left(1+J_{I \mid} \alpha_{l} r_{l}^{\frac{\alpha_{l}-1}{\alpha_{l}}}\right)
\end{array}\right]
$$

To ensure that the system is stable, the product of $\mathbf{M}$ 's eigenvalues $\lambda_{1} \lambda_{2}$, which is equivalent to the determinant of $\mathbf{M}$, has to be positive. In addition, the sum of the two eigenvalues $\lambda_{1}+\lambda_{2}$, which corresponds to $\operatorname{Tr}(\mathbf{M})$, has to be negative. We therefore obtained the following two stability conditions

$$
\begin{aligned}
\lambda_{1} \lambda_{2} & =-\tau_{E}^{-1} \tau_{l}^{-1}\left(J_{E E} \alpha_{E} r_{E}^{\frac{\alpha_{E}-1}{\alpha_{E}}}-1\right)\left(1+J_{l \mid} \alpha_{l} r_{l}^{\frac{\alpha_{l}-1}{\alpha_{l}}}\right)+\tau_{E}^{-1} \tau_{l}^{-1} J_{E I} \alpha_{E} r_{E}^{\frac{\alpha_{E}-1}{\alpha_{E}}} J_{I E} \alpha_{l} r_{l}^{\frac{\alpha_{l}-1}{\alpha_{l}}}>0 \\
\lambda_{1}+\lambda_{2} & =\tau_{E}^{-1}\left(J_{E E \alpha_{E}} r_{E}^{\frac{\alpha_{E}-1}{\alpha_{E}}}-1\right)-\tau_{l}^{-1}\left(1+J_{l \mid} \alpha_{l} r_{l}^{\frac{\alpha_{l}-1}{\alpha_{l}}}\right)<0
\end{aligned}
$$

Notably, the stability conditions depend on the firing rate of the excitatory population $r_{E}$ and the inhibitory population $r_{l}$. Since firing rates are input-dependent, the stability of supralinear networks is input-dependent. In contrast, in linear networks in which $\alpha_{E}=\alpha_{I}=1$, the conditions can be simplified to

$$
\begin{aligned}
\lambda_{1} \lambda_{2} & =-\tau_{E}^{-1} \tau_{I}^{-1}\left(J_{E E}-1\right)\left(1+J_{I I}\right)+\tau_{E}^{-1} \tau_{I}^{-1} J_{E I} J_{I E}>0 \\
\lambda_{1}+\lambda_{2} & =\tau_{E}^{-1}\left(J_{E E}-1\right)-\tau_{I}^{-1}\left(1+J_{I I} \alpha_{l}\right)<0
\end{aligned}
$$

and are thus input-independent.

\section{ISN index for supralinear networks}

If an ensemble is unstable without feedback inhibition, then the ensemble is an ISN (Tsodyks et al., 1997). To determine whether a given system is an ISN, we analyzed the stability of the E-E subnetwork, which is determined by the real part of the leading eigenvalue of the Jacobian of the E-E subnetwork. In the following we call this leading eigenvalue the "ISN index", which is defined as follows:

$$
\text { ISN index }=\tau_{E}^{-1}\left(J_{E E} \alpha_{E} r_{E}^{\frac{\alpha_{E}-1}{\alpha_{E}}}-1\right)
$$


A positive ISN index indicates the system is an ISN. Otherwise, the system is non-ISN. For supralinear networks in which $\alpha_{E}>1$, the ISN index depends on the firing rates, inputs can therefore switch the network from non-ISN to ISN. In contrast, $\alpha_{E}=1$ for linear networks which renders the ISN index firing rate independent. And the ISN index in linear networks solely depends on the recurrent E-E connection strength $J_{E E}$.

\section{Characteristic function}

To investigate how network stability changes with input, we trace the steps of Kraynyukova and Tchumatchenko (2018) and define the characteristic function $F(z)$ as follows:

$$
F(z)=J_{E E}[z]_{+}^{\alpha_{E}}-J_{E I}\left[\operatorname{det} \mathbf{J} \cdot J_{E I}^{-1}[z]_{+}^{\alpha_{E}}+J_{E I}^{-1} J_{I I} z-J_{E I}^{-1} J_{I I} g_{E}+g_{l}\right]_{+}^{\alpha_{I}}-z+g_{E}
$$

where

$$
Z=J_{E E} r_{E}-J_{E I} r_{l}+g_{E}
$$

is the current into the excitatory population. The characteristic function simplifies the original twodimensional system to a one-dimensional system, and the zero crossings of $F(z)$ correspond to the fixed points of the original system. For $z \geq 0$, we note:

$$
\frac{d F(z)}{d z}=J_{E E} \alpha_{E} r_{E}^{\frac{\alpha_{E}-1}{\alpha_{E}}}-J_{E l} \alpha_{l}\left(\operatorname{det} \mathbf{J} \cdot J_{E l}^{-1} \alpha_{E} r_{E}^{\frac{\alpha_{E}-1}{\alpha_{E}}}+J_{E l}^{-1} J_{l l}\right) r_{l}^{\frac{\alpha_{l}-1}{\alpha_{l}}}-1=-\tau_{E} \tau_{l} \lambda_{1} \lambda_{2}
$$

Therefore, if the derivative of $F(z)$ evaluated at one of its roots is positive, the corresponding fixed point is a saddle point. Note that as $r_{E}$ and $r_{l}$ increase, the term in parenthesis becomes dominant. Thus, to ensure that $\lambda_{1} \lambda_{2}$ is negative also for large $r_{E}$ and $r_{l}$, the determinant of the weight matrix det $\mathbf{J}$ has to be positive. Therefore, det $\mathbf{J}$ has a decisive impact on the curvature of $F(z)$. In systems with negative determinant, $F(z)$ bends upwards for large $z$. In contrast, $F(z)$ asymptotically bends downwards in systems with positive determinant. Hence, the high-activity steady-state of systems with negative determinant is unstable. In addition, we can simplify the above condition to the determinant of the weight matrix which is a necessary condition for network stability:

$$
\operatorname{det} \mathbf{J}=-J_{E E} J_{I I}+J_{I E} J_{E I}>0
$$

To investigate how the network stability changes with input $g_{E}$, we examined how $F(z)$ varies with changing input $g_{E}$ by calculating the derivative of $F(z)$ with respect to $g_{E}$,

$$
\frac{d F(z)}{d g_{E}}=\alpha_{J} J_{I I}\left[\operatorname{det} \mathbf{J} \cdot J_{E I}^{-1}[z]_{+}^{\alpha_{E}}+J_{E I}^{-1} J_{I I} z-J_{E l}^{-1} J_{I I} g_{E}+g_{l}\right]_{+}^{\alpha_{l}-1}+1
$$

Since $\frac{d F(z)}{d g_{E}}$ is positive, increasing $g_{E}$ always shifts $F(z)$ upwards, eventually leading to the vanishing of all roots and, thus, unstable dynamics in supralinear networks with negative det $\mathbf{J}$. In 
scenarios in which feedforward input to the inhibitory population also changes, we have

$$
\begin{aligned}
\frac{d F(z)}{d t}= & \frac{\partial F(z)}{\partial g_{E}} \frac{d g_{E}}{d t}+\frac{\partial F(z)}{\partial g_{I}} \frac{d g_{I}}{d t} \\
= & \left(\alpha_{l} J_{I I}\left[\operatorname{det} \mathbf{J} \cdot J_{E I}^{-1}[z]_{+}^{\alpha_{E}}+J_{E I}^{-1} J_{I I} z-J_{E I}^{-1} J_{I I} g_{E}+g_{l}\right]_{+}^{\alpha_{l}-1}+1\right) \Delta g_{E} \\
& -\alpha_{I} J_{E I}\left[\operatorname{det} \mathbf{J} \cdot J_{E I}^{-1}[z]_{+}^{\alpha_{E}}+J_{E I}^{-1} J_{I I} z-J_{E l}^{-1} J_{I I} g_{E}+g_{l}\right]_{+}^{\alpha_{l}-1} \Delta g_{I}
\end{aligned}
$$

When the change in stimulation strength into the excitatory $\left(\Delta g_{E}\right)$ and the inhibitory population $\left(\Delta g_{l}\right)$ are the same, $\frac{d F(z)}{d t}$ is always positive provided $J_{I /}$ is greater than $J_{E l}$. Hence, depending on the value of $\frac{J_{I I}}{J_{E I}}$, stimulation can lead to unstable network dynamics even when the input to the inhibitory population increases more than to the excitatory population.

\section{Spike-frequency adaptation}

We modeled SFA of excitatory neurons as an activity-dependent negative feedback current (Benda and Herz, 2003; Brette and Gerstner, 2005):

$$
\begin{aligned}
\tau_{E} \frac{d r_{E}}{d t} & =-r_{E}+\left[J_{E E} r_{E}-J_{E l} r_{l}+g_{E}\right]_{+}^{\alpha_{E}}-a \\
\tau_{a} \frac{d a}{d t} & =-a+b r_{E}
\end{aligned}
$$

where $a$ is the adaptation variable, $\tau_{a}$ is the adaptation time constant, and $b$ is the adaptation strength.

\section{Short-term plasticity}

We modeled E-to-E STD following previous work (Tsodyks and Markram, 1997; Varela et al., 1997):

$$
\begin{aligned}
\tau_{E} \frac{d r_{E}}{d t} & =-r_{E}+\left[x J_{E E} r_{E}-J_{E I} r_{l}+g_{E}\right]_{+}^{\alpha_{E}} \\
\frac{d x}{d t} & =\frac{1-x}{\tau_{X}}-U_{d} x r_{E}
\end{aligned}
$$

where $x$ is the depression variable, which is limited to the interval $(0,1], \tau_{x}$ is the depression time constant, and $U_{d}$ is the depression rate. The steady-state solution $x^{*}$ of STD is given by

$$
x^{*}=\frac{1}{1+U_{d} r_{E} \tau_{X}}
$$

Similarly, we modeled E-to-I STF as

$$
\begin{aligned}
\tau_{l} \frac{d r_{l}}{d t} & =-r_{l}+\left[u J_{I E} r_{E}-J_{I I} r_{l}+g_{l}\right]_{+}^{\alpha_{l}} \\
\frac{d u}{d t} & =\frac{1-u}{\tau_{u}}+U_{f}\left(U_{\max }-u\right) r_{E}
\end{aligned}
$$


where $u$ is the facilitation variable constrained to the interval $\left[1, U_{\max }\right), U_{\max }$ is the maximal facilitation value, $\tau_{u}$ is the time constant of STF, and $U_{f}$ is the facilitation rate. The steady-state solution $u^{*}$ is given by

$$
u^{*}=\frac{1+U_{f} U_{\max } r_{E} \tau_{u}}{1+U_{f} r_{E} \tau_{u}}
$$

\section{Characteristic function with additional mechanisms}

To visualize how network stability changes in the presence of SFA, we modified the characteristic function by including the adaptation strength $b$. The characteristic function with SFA is given by:

$$
F(z)=J_{E E}[z]_{+}^{\alpha_{E}} /(1+b)-J_{E I}\left[\operatorname{det} \mathbf{J} \cdot J_{E I}^{-1}[z]_{+}^{\alpha_{E}} /(1+b)+J_{E l}^{-1} J_{I I} z-J_{E I}^{-1} J_{I I} g_{E}+g_{I}\right]_{+}^{\alpha_{I}}-z+g_{E}
$$

where

$$
\operatorname{det} \mathbf{J}=-J_{E E} J_{I I}+J_{I E} J_{E I}
$$

Similarly, for networks with E-to-E STD, we can modify the characteristic function by including the depression variable $x$. The characteristic function with E-to-E STD is given by:

$$
F(z)=x J_{E E}[z]_{+}^{\alpha_{E}}-J_{E I}\left[\operatorname{det} \mathbf{J} \cdot J_{E I}^{-1}[z]_{+}^{\alpha_{E}}+J_{E l}^{-1} J_{I I} z-J_{E l}^{-1} J_{I I} g_{E}+g_{l}\right]_{+}^{\alpha_{l}}-z+g_{E}
$$

where

$$
\operatorname{det} \mathbf{J}=-x J_{E E} J_{I I}+J_{I E} J_{E I}
$$

\section{Stability conditions for supralinear networks with additional mechanisms}

To ensure network stability, Equation (10) and the simplified stability condition in Equation (17) have to be satisfied. In the presence of E-to-E STD, this results in the following conditions:

$$
\begin{aligned}
& \operatorname{det} \mathbf{J}=\left|\begin{array}{cc}
x^{*} J_{E E} & -J_{E I} \\
J_{I E} & -J_{I I}
\end{array}\right|=-\frac{1}{1+U_{d} r_{E} \tau_{X}} J_{E E} J_{I I}+J_{I E} J_{E I}>0 \\
& \tau_{I}\left[1-\frac{1}{1+U_{d} r_{E} \tau_{X}} J_{E E} \alpha_{E} r_{E}^{1-\alpha_{E}^{-1}}\right]+\tau_{E}\left[1+J_{I I} \alpha_{I} r_{l}^{1-\alpha_{I}^{-1}}\right]>0
\end{aligned}
$$

In the case of unstable dynamics, $r_{E}$ goes to infinity due to run-away excitation. However, the depression variable $x$ approaches zero in this limit, as $\lim _{r_{E} \rightarrow \infty} x=\lim _{r_{E} \rightarrow \infty} \frac{1}{1+U_{d} r_{E} \tau_{X}}=0$. Therefore, STD terminates excitatory run-away dynamics. As a result, the ensemble transiently becomes an unstable system at stimulus onset. But assuming separation of timescales $\tau_{x} \gg \tau_{E}$, STD ensures that the stability conditions are always satisfied.

In the presence of E-to-I STF, we found a set of similar conditions

$$
\begin{array}{r}
\operatorname{det} \mathbf{J}=\left|\begin{array}{cc}
J_{E E} & -J_{E I} \\
u^{*} J_{I E} & -J_{I I}
\end{array}\right|=-J_{E E} J_{I I}+\frac{1+U_{f} U_{\max } r_{E} \tau_{u}}{1+U_{f} r_{E} \tau_{U}} J_{I E} J_{E I}>0 \\
\tau_{I}\left[1-J_{E E} \alpha_{E} r_{E}^{1-\alpha_{E}^{-1}}\right]+\tau_{E}\left[1+J_{I \mid} \alpha_{I} r_{l}^{1-\alpha_{I}^{-1}}\right]>0
\end{array}
$$


Assuming that $\alpha_{E}=\alpha_{I}=\alpha$, the second condition becomes

$$
\tau_{l}\left[\frac{1}{r_{l}^{1-\alpha^{-1}}}-J_{E E} \alpha\left(\frac{r_{E}}{r_{l}}\right)^{1-\alpha^{-1}}\right]+\tau_{E}\left[\frac{1}{r_{l}^{1-\alpha^{-1}}}+J_{I I} \alpha\right]>0
$$

Substituting the firing rates with the current into excitatory population $z$, we then had

$$
\tau_{l}\left[\frac{1}{r_{I}^{1-\alpha^{-1}}}-J_{E E} \alpha\left(\frac{z}{\operatorname{det} \cdot J_{E I}^{-1}[z]_{+}^{\alpha}+J_{E l}^{-1} J_{I I} z-J_{E I}^{-1} J_{\| I} g_{E}+g_{I}}\right)^{\alpha-1}\right]+\tau_{E}\left[\frac{1}{r_{l}^{1-\alpha^{-1}}+J_{I I} \alpha}>0\right.
$$

Importantly, we can guarantee that the first condition is satisfied by choosing $U_{\max }$ sufficiently large. Since the denominator det $\mathbf{J} \cdot J_{E I}^{-1}[z]_{+}^{\alpha}+J_{E I}^{-1} J_{I I} z-J_{E I}^{-1} J_{I I} g_{E}+g_{I}$ grows faster for $z \gg 1$, the second condition is also satisfied for large $r_{E}$.

In contrast, SFA does not affect the synaptic weights, the determinant of the weight matrix therefore remains negative. Although the system can have two fixed points during stimulation in the presence of weak SFA, the high-activity fixed point is always unstable. Consequently, in the presence of strong adaptation, the system exhibits oscillatory behavior (Fig. S1). To illustrate that SFA induces a limit cycle in networks that would otherwise be unstable, we considered the simplified system without the inhibitory population. The dynamics of the purely excitatory network with SFA are then given as follows:

$$
\begin{aligned}
\tau_{E} \frac{d r_{E}}{d t} & =-r_{E}+\left[J_{E E} r_{E}+g_{E}\right]_{+}^{\alpha_{E}}-a \\
\tau_{a} \frac{d a}{d t} & =-a+b r_{E}
\end{aligned}
$$

The Jacobian of the system is given as

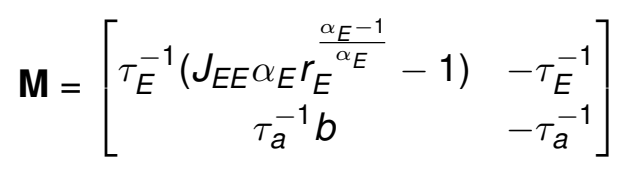

To ensure the stability of the network, the trace of the Jacobian has to be negative

$$
\operatorname{Tr}(\mathbf{M})=\tau_{E}^{-1}\left(J_{E E} \alpha_{E} r_{E}^{\frac{\alpha_{E}-1}{\alpha_{E}}}-1\right)-\tau_{a}^{-1}<0
$$

To satisfy this condition when $J_{E E}$ or $r_{E}$ are large, the time constant $\tau_{a}$ of SFA has to be small. In addition, the determinant of the Jacobian has to be positive

$$
\operatorname{det} \mathbf{M}=-\tau_{a}^{-1} \tau_{E}^{-1}\left(J_{E E} \alpha_{E} r_{E}^{\frac{\alpha_{E}-1}{\alpha_{E}}}-1\right)+\tau_{a}^{-1} \tau_{E}^{-1} b>0
$$

To fulfill this condition, the negative feedback controlled by $b$ has to be strong. However, if both $b$ and $\tau_{a}$ are large, the determinant is positive but the trace switches from negative to positive. As a result, the system undergoes a Hopf bifurcation and exhibits oscillatory behavior (Van Vreeswijk and Hansel, 2001). 


\section{Uni-stability conditions}

The system is said to be "uni-stable", when it has a single stable fixed point. We first identified the uni-stability condition for networks with global inhibition. To that end, we considered a general network with $N$ excitatory populations and $N$ inhibitory populations. To treat this problem analytically, we did not take STP into account in our analysis. The Jacobian matrix of networks with global inhibition $\mathbf{Q}$, can be written as follows,

$$
\mathbf{Q}=\left[\begin{array}{ll}
\mathbf{J}_{E \leftarrow E} & \mathbf{J}_{E \leftarrow I} \\
\mathbf{J}_{/ \leftarrow E} & \mathbf{J}_{/ \leftarrow I}
\end{array}\right]
$$

where $\mathbf{J}_{E \leftarrow E}, \mathbf{J}_{E \leftarrow l}, \mathbf{J}_{/ \leftarrow E}$, and $\mathbf{J}_{/ \leftarrow l}$ are $N$ by $N$ block matrices defined below.

$$
\begin{gathered}
\mathbf{J}_{E \leftarrow E}=\left[\begin{array}{cccc}
a-e & k a & \cdots & k a \\
k a & a-e & \cdots & k a \\
\vdots & \vdots & \ddots & \vdots \\
k a & k a & \cdots & a-e
\end{array}\right] \\
\mathbf{J}_{E \leftarrow I}=-b \mathbf{J}_{N, N} \\
\mathbf{J}_{/ \leftarrow I}=c \mathbf{J}_{N, N} \\
{\left[\begin{array}{cccc}
-d-f & -d & \cdots & -d \\
-d & -d-f & \cdots & -d \\
\vdots & \vdots & \ddots & \vdots \\
-d & -d & \cdots & -d-f
\end{array}\right]}
\end{gathered}
$$

where $a=\tau_{E}^{-1} J_{E E} \alpha_{E}\left[z_{E}\right]_{+}^{\alpha_{E}-1}, b=\tau_{E}^{-1} J_{E I} \alpha_{E}\left[z_{E}\right]_{+}^{\alpha_{E}-1}, c=\tau_{I}^{-1} J_{I E} \alpha_{l}\left[z_{l}\right]_{+}^{\alpha_{l}-1}, d=\tau_{I}^{-1} J_{I I} \alpha_{l}\left[z_{l}\right]_{+}^{\alpha_{I}-1}$, $e=\tau_{E}^{-1}$, and $f=\tau_{I}^{-1}$. Here, $z_{E}$ and $z_{l}$ denote the total current into the excitatory and inhibitory population, respectively. Note that all these parameters are non-negative. Parameter $k$ controls the excitatory connection strength across different populations. $\mathbf{J}_{N, N}$ is a $N$ by $N$ matrix of ones.

The eigenvalues of the Jacobian $\mathbf{Q}$ are roots of its characteristic polynomial,

$$
\operatorname{det}\left(\left(\mathbf{J}_{E \leftarrow E}-\lambda \mathbb{1}\right)\left(\mathbf{J}_{/ \leftarrow I}-\lambda \mathbb{1}\right)-\mathbf{J}_{E \leftarrow /} \mathbf{J}_{/ \leftarrow E}\right)=0
$$

where $\mathbb{1}$ represents the identity matrix of size $N$. The characteristic polynomial can be expanded to:

$$
[(a-e-k a-\lambda)(-f-\lambda)]^{N-1}\left[(a-e-(N-1) k a-\lambda)(-N d-f-\lambda)+N^{2} b c\right]=0
$$


We therefore had four distinct eigenvalues:

$$
\begin{aligned}
& \lambda_{1}=a-e-k a \\
& \lambda_{2}=-f
\end{aligned}
$$

and

$$
\begin{aligned}
\lambda_{3 / 4}= & \frac{1}{2}[(a-e-f-N d+(N-1) k a) \\
& \left. \pm \sqrt{(a-e-f-N d+(N-1) k a)^{2}-4\left((-a f+e f+k a f)-N(a-e) d-N k a f-N(N-1) k a d+N^{2} b c\right)}\right]
\end{aligned}
$$

Note that the eigenvalues $\lambda_{1}$ and $\lambda_{2}$ have an algebraic and geometric multiplicity of $(N-1)$, whereas the eigenvalues $\lambda_{3}$ and $\lambda_{4}$ have an algebraic and geometric multiplicity of 1 .

In analogy to networks with global inhibition, the Jacobian matrix of networks with co-tuned inhibition $\mathbf{R}$, can be written as

$$
\mathbf{R}=\left[\begin{array}{ll}
\mathbf{J}_{E \leftarrow E} & \mathbf{J}_{E \leftarrow I} \\
\mathbf{J}_{/ \leftarrow E} & \mathbf{J}_{/ \leftarrow I}
\end{array}\right]
$$

where $\mathbf{J}_{E \leftarrow E}, \mathbf{J}_{E \leftarrow l}, \mathbf{J}_{/ \leftarrow E}$, and $\mathbf{J}_{/ \leftarrow l}$ are $N$ by $N$ block matrices defined as follows:

$$
\begin{aligned}
& \mathbf{J}_{E \leftarrow E}=\left[\begin{array}{cccc}
a-e & k a & \cdots & k a \\
k a & a-e & \cdots & k a \\
\vdots & \vdots & \ddots & \vdots \\
k a & k a & \cdots & a-e
\end{array}\right] \\
& \mathbf{J}_{E \leftarrow I}=\left[\begin{array}{cccc}
-N b+(N-1) m b & -m b & \cdots & -m b \\
-m b & -N b+(N-1) m b & \cdots & -m b \\
\vdots & \vdots & \ddots & \vdots \\
-m b & -m b & \cdots & -N b+(N-1) m b
\end{array}\right] \\
& \mathbf{J}_{/ \leftarrow E}=\left[\begin{array}{cccc}
N c-(N-1) m c & m c & \cdots & m c \\
m c & N c-(N-1) m c & \cdots & m c \\
\vdots & \vdots & \ddots & \vdots \\
m c & m c & \cdots & N c-(N-1) m c
\end{array}\right] \\
& \mathbf{J}_{/ \leftarrow l}=\left[\begin{array}{cccc}
-N d+(N-1) m d-f & -m d & \cdots & -m d \\
-m d & -N d+(N-1) m d-f & \cdots & -m d \\
\vdots & \vdots & \ddots & \vdots \\
-m d & -m d & \cdots & -N d+(N-1) m d-f
\end{array}\right]
\end{aligned}
$$

where $m$ controls the degree of co-tuning in the network. If $m=0$, the network decouples into $N$ independent ensembles and inhibition is perfectly co-tuned with excitation. In the case $m=1$, inhibition is global and the block matrices become identical to the above case of global inhibition. 
The eigenvalues of the matrix $\mathbf{R}$ are given as the roots of the characteristic polynomial defined by:

$$
\operatorname{det}\left(\left(\mathbf{J}_{E \leftarrow E}-\lambda \mathbb{1}\right)\left(\mathbf{J}_{/ \leftarrow I}-\lambda \mathbb{1}\right)-\mathbf{J}_{E \leftarrow /} \mathbf{J}_{/ \leftarrow E}\right)=0
$$

which yields the following expression:

$$
\left[\lambda^{2}-(a-e-k a-N d+N m d-f) \lambda-(a-e-k a)(N d-N m d-f)+N^{2} b c(1-m)^{2}\right]^{N-1}\left[(a-e-(N-1) k a-\lambda)(-N d-f-\lambda)+N^{2} b c\right]=0
$$

We therefore had four distinct eigenvalues:

$$
\begin{array}{r}
\lambda_{1 / 2}^{\prime}=\frac{1}{2}\left[(a-e-k a-N d+N m d-f) \pm \sqrt{(a-e-k a+N d-N m d+f)^{2}-4 N^{2} b c(1-m)^{2}}\right] \\
\lambda_{3 / 4}^{\prime}=\frac{1}{2}[(a-e-f-N d+(N-1) k a) \\
\left. \pm \sqrt{(a-e-f-N d+(N-1) k a)^{2}-4\left((-a f+e f+k a f)-N(a-e) d-N k a f-N(N-1) k a d+N^{2} b c\right)}\right]
\end{array}
$$

The eigenvalues $\lambda_{1}^{\prime}$ and $\lambda_{2}^{\prime}$ have an algebraic and geometric multiplicity of $(N-1)$, whereas the eigenvalues $\lambda_{3}^{\prime}$ and $\lambda_{4}^{\prime}$ have an algebraic and geometric multiplicity of 1 . We noted that $\lambda_{3}=\lambda_{3}^{\prime}$, $\lambda_{4}=\lambda_{4}^{\prime}$.

To compare under which conditions networks with different structures are uni-stable, we examined the different eigenvalues derived above. As $\lambda_{2}<0$, and $\lambda_{1}^{\prime}>\lambda_{2}^{\prime}$, we only had to compare $\lambda_{1}^{\prime}$ to $\lambda_{1}$. For networks with co-tuned inhibition, we have $m<1$,

$$
\begin{aligned}
\lambda_{1}^{\prime} & =\frac{1}{2}\left[(a-e-k a-N d+N m d-f)+\sqrt{(a-e-k a+N d-N m d+f)^{2}-4 N^{2} b c(1-m)^{2}}\right] \\
& <\frac{1}{2}\left[(a-e-k a-N d+N m d-f)+\sqrt{(a-e-k a+N d-N m d+f)^{2}}\right]=a-e-k a=\lambda_{1}
\end{aligned}
$$

The inequality, $\lambda_{1}^{\prime}<\lambda_{1}$, indicates that networks with co-tuned inhibition have a broad parameter regime in which they are uni-stable than networks with global inhibition. Note that in the absence of a saturating nonlinearity of the input-output function and in the absence of any additional stabilization mechanisms, systems with positive eigenvalues of the Jacobian are unstable. In this case, networks with co-tuned inhibition have a broad parameter regime of being stable than networks with global inhibition.

To visualize the conditions in a two-dimensional plane, we reduced the conditions into a function of $a$ and $d$. For Fig. $3 C, k=0.1, m=0.5$ and $b c=0.9 a d$.

\section{Distance to the decision boundary}

To calculate the distance to the decision boundary in Fig. 4, Fig. 5, Fig. S6 and Fig. S7, we first projected the excitatory activity in Phase 2 onto a two-dimensional Cartesian coordinate system in which the $x$ axis is the activity of the first excitatory ensemble $r_{E 1}$ and the $y$ axis is the activity of the second excitatory ensemble $r_{E 2}$. We then computed the distance between the projected data and the decision boundary which corresponds to the diagonal line in the coordinate system. 


\section{Inhibitory feedback pathways for suppressing unwanted neural activation}

To identify the important neural pathways for the suppression of unwanted neural activation, we analyzed how the activity of the second excitatory ensemble $r_{E 2}$ changes with the input to the first excitatory ensemble $g_{E 1}$. To that end, we considered a general weight matrix for networks with two interacting ensembles

$$
\mathbf{J}=\left[\begin{array}{llll}
J_{E 1 E 1} & J_{E 1 E 2} & -J_{E 1 / 1} & -J_{E 1 / 2} \\
J_{E 2 E 1} & J_{E 2 E 2} & -J_{E 2 / 1} & -J_{E 2 / 2} \\
J_{I 1 E 1} & J_{/ 1 E 2} & -J_{/ 1 / 1} & -J_{/ 1 / 2} \\
J_{I 2 E 1} & J_{I 2 E 2} & -J_{/ 2 / 1} & -J_{I / 2}
\end{array}\right]
$$

We can write the change in firing rate of the excitatory population in the second ensemble $\delta r_{E 2}$ as a function of the change in the input to the other $\delta g_{E 1}$ :

$$
\begin{aligned}
& \delta r_{E 2}=\frac{1}{\operatorname{det}(\mathbb{1}-\mathbf{F J})}\left[\left(-f_{E 2}^{\prime} J_{E 2 E 1}\right) f_{l 1}^{\prime} J_{l 1 / 2} f_{I 2}^{\prime} J_{I 2 / 1}+f_{E 2}^{\prime} J_{E 2 / 1}\left(-f_{/ 1}^{\prime} J_{l 1 E 1}\right)\left(1+f_{I 2}^{\prime} J_{I 2 / 2}\right)\right. \\
& +f_{E 2}^{\prime} J_{E 2 / 2}\left(1+f_{l 1}^{\prime} J_{l 1 / 1}\right)\left(-f_{l 2}^{\prime} J_{l 2 E 1}\right)-\left(-f_{E 2}^{\prime} J_{E 2 E 1}\right)\left(1+f_{l 1}^{\prime} J_{l 1 / 1}\right)\left(1+f_{/ 2}^{\prime} J_{I 2 / 2}\right) \\
& \left.-f_{E 2}^{\prime} J_{E 2 / 1} f_{l 1}^{\prime} J_{l 1 / 2}\left(-f_{l 2}^{\prime} J_{l 2 E 1}\right)-f_{E 2}^{\prime} J_{E 2 / 2}\left(-f_{/ 1}^{\prime} J_{l 1 E 1}\right) f_{l 2}^{\prime} J_{l 2 / 1}\right] f_{E 1}^{\prime} \delta g_{E 1}
\end{aligned}
$$

where $\mathbb{1}$ is the identity matrix. And $\mathbf{F}$ is given by

$$
\mathbf{F}=\left[\begin{array}{cccc}
f_{E 1}^{\prime} & 0 & 0 & 0 \\
0 & f_{E 2}^{\prime} & 0 & 0 \\
0 & 0 & f_{11}^{\prime} & 0 \\
0 & 0 & 0 & f_{12}^{\prime}
\end{array}\right]
$$

where $f_{E 1}^{\prime}, f_{E 2}^{\prime}, f_{l 1}^{\prime}$ and $f_{l 2}^{\prime}$ are the derivatives of the input-output functions evaluated at the fixed point.

Assuming that $J_{E 1 E 1}=J_{E 2 E 2}=J_{E E}, J_{l 1 E 1}=J_{I 2 E 2}=J_{I E}, J_{E 1 / 1}=J_{E 2 / 2}=J_{E I}, J_{l 1 / 1}=J_{I 2 / 2}=J_{I I}$, $J_{E 1 E 2}=J_{E 2 E 1}=J_{E E}^{\prime}, J_{I 1 E 2}=J_{I 2 E 1}=J_{I E}^{\prime}, J_{E 1 / 2}=J_{E 2 / 1}=J_{E I}^{\prime}$ and $J_{l 1 / 2}=J_{I 2 / 1}=J_{I /}^{\prime}$, we find

$$
\begin{array}{r}
\delta r_{E 2}=\frac{1}{\operatorname{det}(\mathbb{1}-\mathbf{F J})}\left[\left(-f_{E 2}^{\prime} J_{E E}^{\prime}\right) f_{I 1}^{\prime} J_{I I}^{\prime} f_{I 2}^{\prime} J_{I I}^{\prime}+f_{E 2}^{\prime} J_{E I}^{\prime}\left(-f_{l 1}^{\prime} J_{I E}\right)\left(1+f_{I 2}^{\prime} J_{I I}\right)\right. \\
+f_{E 2}^{\prime} J_{E I}\left(1+f_{l 1}^{\prime} J_{I I}\right)\left(-f_{I 2}^{\prime} J_{I E}^{\prime}\right)-\left(-f_{E 2}^{\prime} J_{E E}^{\prime}\right)\left(1+f_{l 1}^{\prime} J_{I I}\right)\left(1+f_{I 2}^{\prime} J_{I I}\right) \\
\left.-f_{E 2}^{\prime} J_{E I}^{\prime} f_{l 1}^{\prime} J_{I I}^{\prime}\left(-f_{l 2}^{\prime} J_{I E}^{\prime}\right)-f_{E 2}^{\prime} J_{E I}\left(-f_{l 1}^{\prime} J_{I E}\right) f_{I 2}^{\prime} J_{I I}^{\prime}\right] f_{E 1}^{\prime} \delta g_{E 1}
\end{array}
$$

By further assuming that the weight strengths across ensembles are weak and ignoring the corre- 
sponding higher-order terms, we get

$$
\begin{aligned}
\delta r_{E 2} \approx \frac{1}{\operatorname{det}(\mathbb{1}-\mathbf{F J})}\left[f_{E 2}^{\prime} J_{E I}^{\prime}\left(-f_{l 1}^{\prime} J_{I E}\right)\left(1+f_{I 2}^{\prime} J_{I I}\right)+f_{E 2}^{\prime} J_{E I}\left(1+f_{l 1}^{\prime} J_{I I}\right)\left(-f_{I 2}^{\prime} J_{I E}^{\prime}\right)\right. \\
\left.-\left(-f_{E 2}^{\prime} J_{E E}^{\prime}\right)\left(1+f_{l 1}^{\prime} J_{I I}\right)\left(1+f_{I 2}^{\prime} J_{I I}\right)-f_{E 2}^{\prime} J_{E I}\left(-f_{l 1}^{\prime} J_{I E}\right) f_{I 2}^{\prime} J_{I I}^{\prime}\right] f_{E 1}^{\prime} \delta g_{E 1} \\
=\frac{1}{\operatorname{det}(\mathbb{1}-\mathbf{F J})}\left[\left(\frac{J_{I I}^{\prime}}{J_{E I}^{\prime}} f_{l 2}^{\prime}-\left(\frac{1}{J_{E I}}+f_{I 2}^{\prime} \frac{J_{I I}}{J_{E I}}\right)\right) J_{E I}^{\prime} J_{E I} J_{I E} f_{E 2}^{\prime} f_{l 1}^{\prime}\right. \\
\left.+\left(\frac{J_{E E}^{\prime}}{J_{I E}^{\prime}}\left(1+J_{I I} f_{I 2}^{\prime}\right)-J_{E I} f_{l 2}^{\prime}\right) J_{I E}^{\prime} f_{E 2}^{\prime}\left(1+f_{l 1}^{\prime} J_{I I}\right)\right] f_{E 1}^{\prime} \delta g_{E 1}
\end{aligned}
$$

Note that $\frac{J_{E E}^{\prime}}{J_{I E}^{\prime}}$ and $\frac{J_{I I}^{\prime}}{J_{E I}^{\prime}}$ are terms regulating the respective excitatory and inhibitory input from one ensemble to the excitatory and inhibitory population in another ensemble. The term $\operatorname{det}(\mathbb{1}-\mathbf{F} \mathbf{J})$ is positive to ensure the stability of the system.

To suppress the activity of the excitatory population in the second ensemble $r_{E 2}$, in other words, to ensure that $\delta r_{E 2}<0, J_{I E}^{\prime}$ or/and $J_{E I}^{\prime}$ have to be large. Therefore, we identified $J_{I E}^{\prime}$ and $J_{E I}^{\prime}$ as important synaptic connections which lead to suppression of the unwanted neural activation, suggesting that inhibition can be provided via $J_{I E}^{\prime}$ through the $E 1-/ 2-E 2$ pathway or via $J_{E I}^{\prime}$ through the $E 1-/ 1-E 2$ pathway.

For Fig. 4-5, the rate-based model consists of two ensembles, each of which is composed of 100 excitatory and 25 inhibitory neurons with all-to-all connectivity.

\section{Spiking neural network model}

The spiking neural network model was composed of $N_{E}$ excitatory and $N_{l}$ inhibitory leaky integrateand-fire neurons. Neurons were randomly connected with probability of $20 \%$. The dynamics of membrane potential of neuron $i, U_{i}$, are given by (Zenke et al., 2015):

$$
\tau^{m} \frac{d U_{i}}{d t}=\left(U^{\text {rest }}-U_{i}\right)+g_{i}^{\text {ext }}(t)\left(U^{\text {exc }}-U_{i}\right)+g_{i}^{\text {inh }}(t)\left(U^{\text {inh }}-U_{i}\right)
$$

Here, $\tau^{m}$ is the membrane time constant and $U^{\text {rest }}$ is the resting potential. Spikes are triggered when the membrane potential reaches the spiking threshold $U^{\text {thr }}$. After a spike is emitted, the membrane potential is reset to $U^{\text {rest }}$ and the neuron enters a refractory period of $\tau^{\text {ref }}$. Inhibitory neurons obeyed the same integrate-and-fire formalism but with a shorter membrane time constant.

Excitatory synapses contain a fast AMPA component and a slow NMDA component. The dynamics of the excitatory conductance are described by:

$$
\begin{aligned}
\tau^{\mathrm{ampa}} \frac{d g_{i}^{\mathrm{ampa}}}{d t} & =-g_{i}^{\mathrm{ampa}}+\sum_{j \in \text { exc }} J_{i j} S_{j}(t) \\
\tau^{\mathrm{nmda}} \frac{d g_{i}^{\mathrm{nmda}}}{d t} & =-g_{i}^{\mathrm{nmda}}+g_{i}^{\mathrm{ampa}} \\
g_{i}^{\mathrm{exc}}(t) & =\xi g_{i}^{\mathrm{ampa}}(t)+(1-\xi) g_{i}^{\mathrm{nmda}}(t)
\end{aligned}
$$


Here, $J_{i j}$ denotes the synaptic strength from neuron $j$ to neuron $i$. If the connection does not exist, $J_{i j}$ was set to $0 . S_{j}(t)$ is the spike train of neuron $j$, which is defined as $S_{j}(t)=\sum_{k} \delta\left(t-t_{j}^{k}\right)$, where $\delta$ is the Dirac delta function and $t_{j}^{k}$ the spikes times $k$ of neuron $j$. $\xi$ is a weighting parameter. The dynamics of inhibitory conductances are governed by:

$$
\tau^{\mathrm{gaba}} \frac{d g_{i}^{\mathrm{inh}}}{d t}=-g_{i}^{\mathrm{inh}}+\sum_{j \in \mathrm{inh}} J_{i j} S_{j}(t)
$$

In the spiking neural network models, SFA of excitatory neurons is modeled as follows,

$$
\begin{aligned}
\tau^{m} \frac{d U_{i}}{d t} & =\left(U^{\text {rest }}-U_{i}\right)+g_{i}^{\text {ext }}(t)\left(U^{\text {exc }}-U_{i}\right)+\left(g_{i}^{\text {inh }}(t)+a_{i}(t)\right)\left(U^{\text {inh }}-U_{i}\right) \\
\frac{d}{d t} a_{i}(t) & =-\frac{a_{i}(t)}{\tau_{a}}+b S_{i}(t)
\end{aligned}
$$

where $i$ is the index of excitatory neurons.

The dynamics of E-to-E STD are given by

$$
\begin{aligned}
& \frac{d}{d t} x_{i j}(t)=\frac{1-x_{i j}(t)}{\tau_{x}}-U_{d} x_{i j} S_{j}(t) \\
& \tau^{\mathrm{ampa}} \frac{d g_{i}^{\mathrm{ampa}}}{d t}=-g_{i}^{\mathrm{ampa}}+\sum_{j \in \mathrm{exc}} x_{i j}(t) J_{i j} S_{j}(t)
\end{aligned}
$$

where $i$ represents the index of excitatory neurons.

The dynamics of E-to-I STF are governed by

$$
\begin{aligned}
\frac{d}{d t} u_{i j}(t) & =\frac{1-u_{i j}(t)}{\tau_{u}}+U_{f}\left(U_{m a x}-u_{i j}(t)\right) S_{j}(t) \\
\tau^{\mathrm{ampa}} \frac{d g_{i}^{\mathrm{ampa}}}{d t} & =-g_{i}^{\mathrm{ampa}}+\sum_{j \in \mathrm{exc}} u_{i j}(t) J_{i j} S_{j}(t)
\end{aligned}
$$

where $i$ denotes the index of inhibitory neurons.

For Fig. 6, each excitatory and inhibitory neuron received external excitatory input from 300 neurons firing with Poisson statistics at an average firing rate of $0.1 \mathrm{~Hz}$ at baseline. During stimulation, the excitatory neurons corresponding to the activated pattern received external excitatory input from 300 neurons firing with Poisson statistics at an average firing rate of $0.5 \mathrm{~Hz}$. The pattern activity with each stored pattern is quantified by the dot product of the neural activity with the stored pattern. And neural activity is computed by the instantaneous firing rates with $10 \mathrm{~ms}$ bin size. The difference in pattern activity for the peak amplitude is calculated by subtracting the average maximal pattern activity of the unstimulated patterns from the maximal pattern activity of the activated pattern. Similarly, the difference in pattern activity for the fixed point is calculated by subtracting the average pattern activity of the unstimulated patterns at the fixed point from the pattern activity 
of the activated pattern at the fixed point. Fixed point activity is computed by averaging the activity of the middle 1 second within the 2 -second stimulation period.

For Fig. S5, each excitatory and inhibitory neuron received external excitatory input from 300 neurons firing with Poisson statistics at an average firing rate of $0.1 \mathrm{~Hz}$ at the baseline. During stimulation, each excitatory neuron received external excitatory input from 300 neurons firing with Poisson statistics at an average firing rate of $0.3 \mathrm{~Hz}$.

\section{Simulations}

Simulations were performed in Python and Mathematica. All differential equations were implemented by Euler integration with a time step of $0.1 \mathrm{~ms}$. All simulation parameters are listed in Tables 1-7. The simulation source code to reproduce the figures is publicly available at https: //github.com/fmi-basel/gzenke-nonlinear-transient-amplification.

\begin{tabular}{lccl}
\hline & \multicolumn{3}{c}{ Table 1: Parameters for Figure 1C-E } \\
\hline Symbol & Value & Unit & Description \\
\hline$J_{E E}$ & 1.8 & - & E-to-E connection strength \\
$J_{I E}$ & 1.0 & - & E-to-I connection strength \\
$J_{E I}$ & 1.0 & - & I-to-E connection strength \\
$J_{I I}$ & 0.6 & - & I-to-I connection strength \\
$\alpha_{E}$ & 2 & - & power of excitatory input-output function \\
$\alpha_{I}$ & 2 & - & power of inhibitory input-output function \\
$\tau_{E}$ & 20 & ms & time constant of excitatory firing dynamics \\
$\tau_{I}$ & 10 & ms & time constant of inhibitory firing dynamics \\
$g_{E}^{b s}$ & 1.55 & - & input to the $E$ population at baseline \\
$g_{E}^{s t i m}$ & 3.0 & - & input to the $E$ population during stimulation \\
$g_{I}$ & 2.0 & - & input to the I population \\
\hline & 1.5 & Parameters for Figure 1F \\
\hline$J_{I E}$ & & - & E-to-I connection strength \\
$J_{E I}$ & 0.45 & I-to-E connection strength \\
$J_{I I}$ & 1.0 & I-to-I connection strength
\end{tabular}


Table 2: Parameters for Figure 2

\begin{tabular}{lccl}
\hline Symbol & Value & Unit & Description \\
\hline$\tau_{a}$ & 200 & $\mathrm{~ms}$ & time constant of SFA \\
$b$ & 1.0 & - & strength of SFA \\
$\tau_{x}$ & 200 & $\mathrm{~ms}$ & time constant of STD \\
$U_{d}$ & 1.0 & - & depression rate \\
$\tau_{u}$ & 200 & $\mathrm{~ms}$ & time constant of STF \\
$U_{f}$ & 1.0 & - & facilitation rate \\
$U_{\max }$ & 6.0 & - & maximal facilitation value \\
\hline
\end{tabular}

Note that these values are also applied elsewhere unless mentioned otherwise.

Table 3: Parameters for Figure 3 bi/multi-stable example

\begin{tabular}{lccl}
\hline Symbol & Value & Unit & Description \\
\hline$J_{E E}$ & 1.4 & - & within-ensemble E-to-E connection strength \\
$J_{I E}$ & 0.6 & - & within-ensemble E-to-I connection strength \\
$J_{E I}$ & 1.0 & - & within-ensemble I-to-E connection strength \\
$J_{I I}$ & 0.6 & - & within-ensemble I-to-I connection strength \\
$J_{E E}^{\prime}$ & 0.14 & - & inter-ensemble E-to-E connection strength \\
$J_{I E}^{\prime}$ & 0.6 & - & inter-ensemble E-to-I connection strength \\
$J_{E I}^{\prime}$ & 1.0 & - & inter-ensemble I-to-E connection strength \\
$J_{I I}^{\prime}$ & 0.6 & - & inter-ensemble I-to-I connection strength \\
$g_{E 1}^{b S}$ & 2.2 & - & input to the $E 1$ population at baseline \\
$g_{E 1}^{s t i m}$ & 3.0 & - & input to the E1 population during stimulation \\
$g_{E 2}$ & 2.2 & - & input to the E2 population \\
$g_{I}$ & 2.0 & - & input to the / population \\
\hline & 1.3 & Parameters for Figure 3 uni-stable example \\
\hline$J_{E E}$ & 0.13 & - & within-ensemble E-to-E connection strength \\
$J_{E E}^{\prime}$ & & inter-ensemble E-to-E connection strength
\end{tabular}


Table 4: Parameters for Figure 4-5

\begin{tabular}{|c|c|c|c|}
\hline Symbol & Value & Unit & Description \\
\hline$N_{E}$ & 200 & - & number of excitatory neurons \\
\hline$N_{l}$ & 50 & - & number of inhibitory neurons \\
\hline$N$ & 2 & - & number of ensembles \\
\hline$J_{E E}$ & $1.2 /\left(N_{E} / 2-1\right)$ & - & within-ensemble E-to-E connection strength \\
\hline$J_{I E}$ & $1.0 /\left(N_{E} / 2\right)$ & - & within-ensemble E-to-I connection strength \\
\hline$J_{E I}$ & $1.0 /\left(N_{l} / 2\right)$ & - & within-ensemble I-to-E connection strength \\
\hline$J_{\| I}$ & $1.0 /\left(N_{l} / 2-1\right)$ & - & within-ensemble I-to-I connection strength \\
\hline$J_{E E}^{\prime}$ & $0.36 /\left(N_{E} / 2-1\right)$ & - & inter-ensemble E-to-E connection strength \\
\hline$J_{I E}^{\prime}$ & $0.4 /\left(N_{E} / 2\right)$ & - & inter-ensemble E-to-I connection strength \\
\hline$J_{E I}^{\prime}$ & $0.1 /\left(N_{l} / 2\right)$ & - & inter-ensemble I-to-E connection strength \\
\hline$J_{\| I}^{\prime}$ & $0.1 /\left(N_{l} / 2\right)$ & - & inter-ensemble I-to-I connection strength \\
\hline$g_{l}$ & 2.0 & - & input to the I population \\
\hline \multicolumn{4}{|c|}{ Parameters for Figure 4} \\
\hline$g_{E 1}^{b s}$ & 1.35 & - & input to the $E 1$ population at baseline \\
\hline$g_{E 1}^{s t i m}$ & 4.0 & - & input to the $E 1$ population during stimulation \\
\hline$g_{E 2}$ & 1.35 & - & input to the $E 2$ population \\
\hline \multicolumn{4}{|c|}{ Parameters for Figure 5} \\
\hline$g_{E 1}^{b s}$ & 1.35 & - & input to the $E 1$ population at baseline \\
\hline$g_{E 1}^{\text {stim }}$ & $1.35+(4.0-1.35)(1-p)$ & - & input to the $E 1$ population during stimulation \\
\hline$g_{E 2}^{b s}$ & 1.35 & - & input to the $E 2$ population at baseline \\
\hline$g_{E 2}^{s t i m}$ & $1.35+(4.0-1.35) p$ & - & input to the $E 2$ population during stimulation \\
\hline
\end{tabular}

Here, $p$ is a parameter between 0 and 1 controlling the additional inputs to $E 1$ and $E 2$. 


\begin{tabular}{|c|c|c|c|}
\hline \multicolumn{4}{|c|}{ Table 5: Parameters for Figure 6} \\
\hline Symbol & Value & Unit & Description \\
\hline$N_{E}$ & 400 & - & number of excitatory neurons \\
\hline$N_{l}$ & 100 & - & number of inhibitory neurons \\
\hline$U^{\text {rest }}$ & -70 & $\mathrm{mV}$ & resting membrane potential \\
\hline$U^{\mathrm{exc}}$ & 0 & $\mathrm{mV}$ & excitatory reversal potential \\
\hline$U^{\mathrm{inh}}$ & -80 & $\mathrm{mV}$ & inhibitory reversal potential \\
\hline$\tau^{\text {ref }}$ & 3 & $\mathrm{~ms}$ & duration of refractory period \\
\hline$\tau_{\mathrm{exc}}^{m}$ & 20 & $\mathrm{~ms}$ & membrane time constant of excitatory neurons \\
\hline$\tau_{\text {inh }}^{m}$ & 10 & $\mathrm{~ms}$ & membrane time constant of inhibitory neurons \\
\hline$\tau^{\text {ampa }}$ & 5 & $\mathrm{~ms}$ & time constant of AMPA receptor \\
\hline$\tau^{\text {gaba }}$ & 10 & $\mathrm{~ms}$ & time constant of GABA receptor \\
\hline$\tau^{\mathrm{nmda}}$ & 100 & $\mathrm{~ms}$ & time constant of NMDA receptor \\
\hline$\xi$ & 0.5 & - & receptor weighting factor \\
\hline$J_{E E}$ & 0.19 & - & within-ensemble E-to-E connection strength \\
\hline$J_{I E}$ & 0.10 & - & within-ensemble E-to-I connection strength \\
\hline$J_{E I}$ & 0.10 & - & within-ensemble I-to-E connection strength \\
\hline$J_{\|}$ & 0.06 & - & within-ensemble I-to-I connection strength \\
\hline$J_{E E}^{\prime}$ & 0.019 & - & inter-ensemble E-to-E connection strength \\
\hline$J_{I E}^{\prime}$ & 0.05 & - & inter-ensemble E-to-I connection strength \\
\hline$J_{E I}^{\prime}$ & 0.04 & - & inter-ensemble I-to-E connection strength \\
\hline$J_{\| \prime}^{\prime}$ & 0.006 & - & inter-ensemble I-to-I connection strength \\
\hline
\end{tabular}

Table 6: Parameters for Figure S5

\begin{tabular}{lccl}
\hline Symbol & Value & Unit & Description \\
\hline$N_{E}$ & 400 & - & number of excitatory neurons \\
$N_{I}$ & 100 & - & number of inhibitory neurons \\
$J_{E E}$ & 0.05 & - & E-to-E connection strength \\
$J_{I E}$ & 0.02 & - & E-to-I connection strength \\
$J_{E I}$ & 0.05 & - & I-to-E connection strength \\
$J_{I I}$ & 0.03 & - & I-to-I connection strength
\end{tabular}

Table 7: Parameters for Figure S9

\begin{tabular}{lccl}
\hline Symbol & Value & Unit & Description \\
\hline$J_{E E}$ & 0.5 & - & E-to-E connection strength \\
$J_{I E}$ & 0.45 & - & E-to-I connection strength \\
$J_{E I}$ & 1.0 & - & I-to-E connection strength \\
$J_{I I}$ & 1.5 & - & I-to-I connection strength \\
$g_{E}^{b S}$ & 0.5 & - & input to the $E$ population at baseline \\
$g_{I}^{b S}$ & 1.5 & - & input to the / population at baseline
\end{tabular}




\section{Acknowledgments}

We thank Rainer W. Friedrich, Claire Meissner-Bernard, William F. Podlaski, and members of the Zenke Group for comments and discussions. This work was supported by the Novartis Research Foundation.

\section{Author contributions}

Y.K.W. and F.Z. conceived the study. Y.K.W. performed model analyses and simulations. Y.K.W. and F.Z. wrote the manuscript.

\section{Competing interests}

The authors declare no competing interests.

\section{References}

Ahmadian Y, Rubin DB, Miller KD. Analysis of the stabilized supralinear network. Neural Computation 2013;25(8):1994-2037. doi: https://doi.org/10.1162/NECO_a_00472.

Amit DJ, Brunel N. Model of global spontaneous activity and local structured activity during delay periods in the cerebral cortex. Cerebral Cortex 1997;7(3):237-252. doi: https://doi.org/10.1093/ cercor/7.3.237.

Baker C, Zhu V, Rosenbaum R. Nonlinear stimulus representations in neural circuits with approximate excitatory-inhibitory balance. PLoS Computational Biology 2020;16(9):1-30. doi: https://doi.org/10.1371/journal.pcbi.1008192.

Barkai E, Hasselmo ME. Modulation of the input/output function of rat piriform cortex pyramidal cells. Journal of Neurophysiology 1994;72(2):644-658. doi: https://doi.org/10.1152/jn.1994.72. 2.644 .

Benda J, Herz AVM. A universal model for spike-frequency adaptation. Neural Computation 2003;15(11):2523-2564. doi: https://doi.org/10.1162/089976603322385063.

Bissieére S, Humeau Y, Luüthi A. Dopamine gates LTP induction in lateral amygdala by suppressing feedforward inhibition. Nature Neuroscience 2003;6(6):587-592. doi: https://doi.org/ 10.1038/nn1058.

Bolding KA, Franks KM. Recurrent cortical circuits implement concentration-invariant odor coding. Science 2018;361(6407). doi: https://doi.org/10.1126/science.aat6904. 
Bondanelli G, Ostojic S. Coding with transient trajectories in recurrent neural networks. PLoS Computational Biology 2020;16(2):1-36. doi: https://doi.org/10.1371/journal.pcbi.1007655.

Brette R, Gerstner W. Adaptive exponential integrate-and-fire model as an effective description of neuronal activity. Journal of Neurophysiology 2005;94(5):3637-3642. doi: https://doi.org/10. 1152/jn.00686.2005.

Christodoulou G, Vogels TP, Agnes EJ. Regimes and mechanisms of transient amplification in abstract and biological networks. bioRxiv 2021; doi: https://doi.org/10.1101/2021.04.01.437964.

Churchland MM, Cunningham JP, Kaufman MT, Foster JD, Nuyujukian P, Ryu SI, et al. Neural population dynamics during reaching. Nature 2012;487(7405):51-56. doi: https://doi.org/10. 1038/nature11129.

Compte A, Brunel N, Goldman-Rakic PS, Wang XJ. Synaptic mechanisms and network dynamics underlying spatial working memory in a cortical network model. Cerebral Cortex 2000;10(9):910-923. doi: https://doi.org/10.1093/cercor/10.9.910.

Cossell L, lacaruso MF, Muir DR, Houlton R, Sader EN, Ko H, et al. Functional organization of excitatory synaptic strength in primary visual cortex. Nature 2015;518(7539):399-403. doi: https://doi.org/10.1038/nature14182.

Cruikshank SJ, Lewis TJ, Connors BW. Synaptic basis for intense thalamocortical activation of feedforward inhibitory cells in neocortex. Nature Neuroscience 2007;10(4):462-468. doi: https: //doi.org/10.1038/nn1861.

Denève S, Machens CK. Efficient codes and balanced networks. Nature Neuroscience 2016;19(3):375-382. doi: https://doi.org/10.1038/nn.4243.

DeWeese MR, Wehr M, Zador AM. Binary spiking in auditory cortex. Journal of Neuroscience 2003;23(21):7940-7949. doi: https://doi.org/10.1523/JNEUROSCI.23-21-07940.2003.

El-Gaby M, Reeve HM, Lopes-dos Santos V, Campo-Urriza N, Perestenko PV, Morley A, et al. An emergent neural coactivity code for dynamic memory. Nature Neuroscience $2021 ; 24(5)$ :694704. doi: https://doi.org/10.1038/s41593-021-00820-w.

Engel AK, Fries P, Singer W. Dynamic predictions: Oscillations and synchrony in top-down processing. Nature Reviews Neuroscience 2001;2(10):704-716. doi: https://doi.org/10.1038/ 35094565.

Ermentrout B. Linearization of F-I Curves by Adaptation. Neural Computation 1998;10(7):17211729. doi: https://doi.org/10.1162/089976698300017106.

Fairhall AL, Lewen GD, Bialek W, de Ruyter van Steveninck RR. Efficiency and ambiguity in an adaptive neural code. Nature 2001;412(6849):787-792. doi: https://doi.org/10.1038/35090500. 
Franks KM, Russo MJ, Sosulski DL, Mulligan AA, Siegelbaum SA, Axel R. Recurrent circuitry dynamically shapes the activation of piriform cortex. Neuron 2011;72(1):49-56. doi: https: //doi.org/10.1016/j.neuron.2011.08.020.

Freedman DJ, Riesenhuber M, Poggio T, Miller EK. Categorical representation of visual stimuli in the primate prefrontal cortex. Science 2001;291(5502):312-316. doi: https://doi.org/10.1126/ science.291.5502.312.

Froemke RC, Merzenich MM, Schreiner CE. A synaptic memory trace for cortical receptive field plasticity. Nature 2007;450(7168):425-429. doi: https://doi.org/10.1038/nature06289.

Funahashi S, Bruce CJ, Goldman-Rakic PS. Mnemonic coding of visual space in the monkey's dorsolateral prefrontal cortex. Journal of Neurophysiology 1989;61(2):331-349. doi: https: //doi.org/10.1152/jn.1989.61.2.331.

Gillary G, von der Heydt R, Niebur E. Short-term depression and transient memory in sensory cortex. Journal of Computational Neuroscience 2017;43(3):273-294. doi: https://doi.org/10. 1007/s10827-017-0662-8.

Gillett M, Pereira U, Brunel N. Characteristics of sequential activity in networks with temporally asymmetric Hebbian learning. Proceedings of the National Academy of Sciences of the United States of America 2020;117(47):29948-29958. doi: https://doi.org/10.1073/pnas.1918674117.

Gjoni E, Zenke F, Bouhours B, Schneggenburger R. Specific synaptic input strengths determine the computational properties of excitation-inhibition integration in a sound localization circuit. Journal of Physiology 2018;596(20):4945-4967. doi: https://doi.org/10.1113/JP276012.

Goldman MS. Memory without feedback in a neural network. Neuron 2009;61(4):621-634. doi: https://doi.org/10.1016/j.neuron.2008.12.012.

Harris KD. Neural signatures of cell assembly organization. Nature Reviews Neuroscience 2005;6(5):399-407. doi: https://doi.org/10.1038/nrn1669.

Harris KD, Csicsvari J, Hirase H, Dragoi G, Buzsáki G. Organization of cell assemblies in the hippocampus. Nature 2003;424(6948):552-556. doi: https://doi.org/10.1038/nature01834.

Hebb DO. The organization of behavior: a neuropsychological theory. Wiley, New York; 1949. doi: https://doi.org/10.4324/9781410612403.

Hempel CM, Hartman KH, Wang XJ, Turrigiano GG, Nelson SB. Multiple forms of short-term plasticity at excitatory synapses in rat medial prefrontal cortex. Journal of Neurophysiology 2000;83(5):3031-3041. doi: https://doi.org/10.1152/jn.2000.83.5.3031. 
Hennequin G, Agnes EJ, Vogels TP. Inhibitory plasticity: Balance, control, and codependence. Annual Review of Neuroscience 2017;40:557-579. doi: https://doi.org/10.1146/ annurev-neuro-072116-031005.

Hennequin G, Ahmadian Y, Rubin DB, Lengyel M, Miller KD. The dynamical regime of sensory cortex: Stable dynamics around a single stimulus-tuned attractor account for patterns of noise variability. Neuron 2018;98(4):846-860.e5. doi: https://doi.org/10.1016/j.neuron.2018.04.017.

Hennequin G, Vogels TP, Gerstner W. Non-normal amplification in random balanced neuronal networks. Physical Review E - Statistical, Nonlinear, and Soft Matter Physics 2012;86(1):1-12. doi: https://doi.org/10.1103/PhysRevE.86.011909.

Hennequin G, Vogels TP, Gerstner W. Optimal control of transient dynamics in balanced networks supports generation of complex movements. Neuron 2014;82(6):1394-1406. doi: https://doi. org/10.1016/j.neuron.2014.04.045.

Hopfield JJ. Neural networks and physical systems with emergent collective computational abilities. Proceedings of the national academy of sciences 1982;79(8):2554-2558. doi: https: //doi.org/10.1073/pnas.79.8.2554.

Ji XY, Zingg B, Mesik L, Xiao Z, Zhang LI, Tao HW. Thalamocortical innervation pattern in mouse auditory and visual cortex: Laminar and cell-type specificity. Cerebral Cortex 2016;26(6):26122625. doi: https://doi.org/10.1093/cercor/bhv099.

Josselyn SA, Tonegawa S. Memory engrams: Recalling the past and imagining the future. Science 2020;367(6473). doi: https://doi.org/10.1126/science.aaw4325.

Ko H, Hofer SB, Pichler B, Buchanan KA, Sjöström PJ, Mrsic-Flogel TD. Functional specificity of local synaptic connections in neocortical networks. Nature 2011;473(7345):87-91. doi: https: //doi.org/10.1038/nature09880.

Kraynyukova N, Tchumatchenko T. Stabilized supralinear network can give rise to bistable, oscillatory, and persistent activity. Proceedings of the National Academy of Sciences of the United States of America 2018;115(13):3464-3469. doi: https://doi.org/10.1073/pnas.1700080115.

Large AM, Vogler NW, Mielo S, Oswald AMM. Balanced feedforward inhibition and dominant recurrent inhibition in olfactory cortex. Proceedings of the National Academy of Sciences of the United States of America 2016;113(8):2276-2281. doi: https://doi.org/10.1073/pnas.1519295113.

Levina A, Herrmann JM, Geisel T. Dynamical synapses causing self-organized criticality in neural networks. Nature Physics 2007;3(12):857-860. doi: https://doi.org/10.1038/nphys758.

Litwin-Kumar A, Doiron B. Slow dynamics and high variability in balanced cortical networks with clustered connections. Nature Neuroscience 2012;15(11):1498-1505. doi: https://doi.org/10. 1038/nn.3220. 
Markram H, Wang Y, Tsodyks M. Differential signaling via the same axon of neocortical pyramidal neurons. Proceedings of the National Academy of Sciences of the United States of America 1998;95(9):5323-5328. doi: https://doi.org/10.1073/pnas.95.9.5323.

Marshel JH, Kim YS, Machado TA, Quirin S, Benson B, Kadmon J, et al. Cortical layer-specific critical dynamics triggering perception. Science 2019;365(6453). doi: https://doi.org/10.1126/ science.aaw5202.

Mazor O, Laurent G. Transient dynamics versus fixed points in odor representations by locust antennal lobe projection neurons. Neuron 2005;48(4):661-673. doi: https://doi.org/10.1016/j. neuron.2005.09.032.

Mazzucato L, La Camera G, Fontanini A. Expectation-induced modulation of metastable activity underlies faster coding of sensory stimuli. Nature Neuroscience 2019;22(5):787-796. doi: https: //doi.org/10.1038/s41593-019-0364-9.

Miller KD, Palmigiano A. Generalized paradoxical effects in excitatory/inhibitory networks. bioRxiv 2020; doi: https://doi.org/10.1101/2020.10.13.336727.

Miska NJ, Richter LMA, Cary BA, Gjorgjieva J, Turrigiano GG. Sensory experience inversely regulates feedforward and feedback excitation-inhibition ratio in rodent visual cortex. eLife 2018;7:127. doi: https://doi.org/10.7554/eLife.38846.

Mongillo G, Barak O, Tsodyks M. Synaptic theory of working memory. Science 2008;319(5869):1543-1546. doi: https://doi.org/10.1126/science.1150769.

Mongillo G, Hansel D, van Vreeswijk C. Bistability and Spatiotemporal Irregularity in Neuronal Networks with Nonlinear Synaptic Transmission. Physical Review Letters 2012 Apr;108(15):158101. doi: https://doi.org/10.1103/PhysRevLett.108.158101.

Murphy BK, Miller KD. Balanced amplification: A new mechanism of selective amplification of neural activity patterns. Neuron 2009;61(4):635-648. doi: https://doi.org/10.1016/j.neuron.2009.02. 005.

Niessing J, Friedrich RW. Olfactory pattern classification by discrete neuronal network states. Nature 2010;465(7294):47-52. doi: https://doi.org/10.1038/nature08961.

Okun M, Lampl I. Instantaneous correlation of excitation and inhibition during ongoing and sensory-evoked activities. Nature Neuroscience 2008;11(5):535-537. doi: https://doi.org/10. 1038/nn.2105.

Pala $\mathrm{A}$, Petersen $\mathrm{CCH}$. In vivo measurement of cell-type-specific synaptic connectivity and synaptic transmission in layer 2/3 mouse barrel cortex. Neuron 2015;85(1):68-75. doi: https://doi.org/10.1016/j.neuron.2014.11.025. 
Peron S, Pancholi R, Voelcker B, Wittenbach JD, Ólafsdóttir HF, Freeman J, et al. Recurrent interactions in local cortical circuits. Nature 2020;579(7798):256-259. doi: https://doi.org/10. 1038/s41586-020-2062-x.

Ponce-Alvarez A, Thiele A, Albright TD, Stoner GR, Deco G. Stimulus-dependent variability and noise correlations in cortical MT neurons. Proceedings of the National Academy of Sciences of the United States of America 2013;110(32):13162-13167. doi: https://doi.org/10.1073/pnas. 1300098110.

Pozzorini C, Naud R, Mensi S, Gerstner W. Temporal whitening by power-law adaptation in neocortical neurons. Nature Neuroscience 2013;16(7):942-948. doi: https://doi.org/10.1038/nn.3431.

Priebe NJ, Mechler F, Carandini M, Ferster D. The contribution of spike threshold to the dichotomy of cortical simple and complex cells. Nature Neuroscience 2004;7(10):1113-1122. doi: https: //doi.org/10.1038/nn1310.

Romo R, Brody CD, Hernández A, Lemus L. Neuronal correlates of parametric working memory in the prefrontal cortex. Nature 1999;399(6735):470-473. doi: https://doi.org/10.1038/20939.

Rost T, Deger M, Nawrot MP. Winnerless competition in clustered balanced networks: Inhibitory assemblies do the trick. Biological Cybernetics 2018;112(1):81-98. doi: https://doi.org/10.1007/ s00422-017-0737-7.

Rubin DB, VanHooser SD, Miller KD. The stabilized supralinear network: A unifying circuit motif underlying multi-input integration in sensory cortex. Neuron 2015;85(2):402-417. doi: https: //doi.org/10.1016/j.neuron.2014.12.026.

Rubin R, Abbott LF, Sompolinsky H. Balanced excitation and inhibition are required for highcapacity, noise-robust neuronal selectivity. Proceedings of the National Academy of Sciences of the United States of America 2017;114(44):E9366-E9375. doi: https://doi.org/10.1073/pnas. 1705841114.

Rupprecht P, Friedrich RW. Precise synaptic balance in the zebrafish homolog of olfactory cortex. Neuron 2018;100(3):669-683.e5. doi: https://doi.org/10.1016/j.neuron.2018.09.013.

Sanzeni A, Akitake B, Goldbach HC, Leedy CE, Brunel N, Histed MH. Inhibition stabilization is a widespread property of cortical networks. eLife 2020;9:1-39. doi: https://doi.org/10.7554/eLife. 54875.

Schulz A, Miehl C, Berry MJ, Gjorgjieva J. The generation of cortical novelty responses through inhibitory plasticity. bioRxiv 2020; doi: https://doi.org/10.1101/2020.11.30.403840.

Seeholzer A, Deger M, Gerstner W. Stability of working memory in continuous attractor networks under the control of short-term plasticity. PLoS Computational Biology 2019;15(4):1-48. doi: https://doi.org/10.1371/journal.pcbi.1006928. 
Shew WL, Clawson WP, Pobst J, Karimipanah Y, Wright NC, Wessel R. Adaptation to sensory input tunes visual cortex to criticality. Nature Physics 2015;11(8):659-663. doi: https://doi.org/ 10.1038/nphys3370.

Stopfer M, Bhagavan S, Smith BH, Laurent G. Normalization for sparse encoding of odours by a wide-filed interneurons. Nature 1997;390(6655):70-74. doi: https://doi.org/10.1126/science. 1201835.

Thorpe S, Fize D, Marlot C. Speed of processing in the human visual system. Nature 1996;381(6582):520-522. doi: https://doi.org/10.1038/381520a0.

Tsodyks MV, Markram H. The neural code between neocortical pyramidal neurons depends on neurotransmitter release probability. Proceedings of the National Academy of Sciences of the United States of America 1997;94(2):719-723. doi: https://doi.org/10.1073/pnas.94.2.719.

Tsodyks MV, Skaggs WE, Sejnowski TJ, McNaughton BL. Paradoxical effects of external modulation of inhibitory interneurons. Journal of Neuroscience 1997;17(11):4382-4388. doi: https://doi.org/10.1523/jneurosci.17-11-04382.1997.

Van Vreeswijk C, Hansel D. Patterns of synchrony in neural networks with spike adaptation. Neural Computation 2001;13(5):959-992. doi: https://doi.org/10.1162/08997660151134280.

Van Vreeswijk C, Sompolinsky H. Chaos in neuronal networks with balanced excitatory and inhibitory activity. Science 1996;274(5293):1724-1726. doi: https://doi.org/10.1126/science.274. 5293.1724.

Varela JA, Sen K, Gibson J, Fost J, Abbott LF, Nelson SB. A quantitative description of short-term plasticity at excitatory synapses in layer $2 / 3$ of rat primary visual cortex. Journal of Neuroscience 1997;17(20):7926-7940. doi: https://doi.org/10.1523/jneurosci.17-20-07926.1997.

Vogels TP, Sprekeler H, Zenke F, Clopath C, Gerstner W. Inhibitory plasticity balances excitation and inhibition in sensory pathways and memory networks. Science 2011;334(6062):1569-1573. doi: https://doi.org/10.1126/science.1211095.

Wehr M, Zador AM. Balanced inhibition underlies tuning and sharpens spike timing in auditory cortex. Nature 2003;426(6965):442-446. doi: https://doi.org/10.1038/nature02116.

Wong KF, Wang XJ. A recurrent network mechanism of time integration in perceptual decisions. Journal of Neuroscience 2006;26(4):1314-1328. doi: https://doi.org/10.1523/JNEUROSCI. 3733-05.2006.

Wu YK, Hengen KB, Turrigiano GG, Gjorgjieva J. Homeostatic mechanisms regulate distinct aspects of cortical circuit dynamics. Proceedings of the National Academy of Sciences of 
the United States of America 2020;117(39):24514-24525. doi: https://doi.org/10.1073/pnas. 1918368117.

Yakovlev V, Fusi S, Herman E, Zohary E. Inter-trial neuronal activity in inferior temporal cortex: A putative vehicle to generate long-term visual associations. Nature Neuroscience 1998;1(4):310317. doi: https://doi.org/10.1038/1131.

Zenke F, Agnes EJ, Gerstner W. Diverse synaptic plasticity mechanisms orchestrated to form and retrieve memories in spiking neural networks. Nature Communications 2015;6(1):1-13. doi: https://doi.org/10.1038/ncomms7922.

Znamenskiy P, Kim MH, Muir DR, lacaruso MF, Hofer SB, Mrsic-Flogel TD. Functional selectivity and specific connectivity of inhibitory neurons in primary visual cortex. bioRxiv 2018; doi: https: //doi.org/10.1101/294835.

Zucker RS, Regehr WG. Short-term synaptic plasticity. Annual Review of Physiology 2002;64:355-405. doi: https://doi.org/10.1146/annurev.physiol.64.092501.114547. 


\section{Supplementary Figures}

A

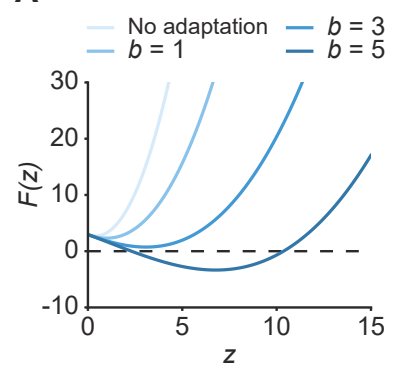

B

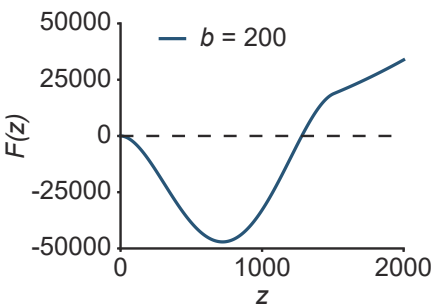

C

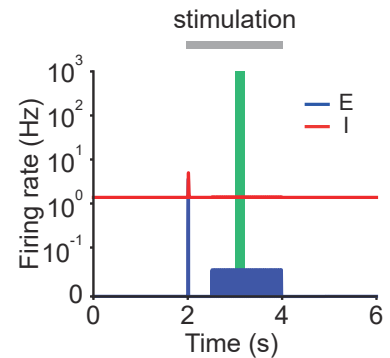

strong spike-frequency adaptation

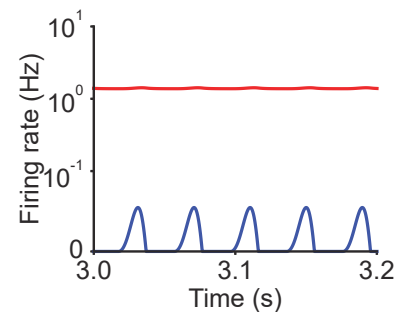

Fig. S1. (A) Characteristic function $F(z)$ in networks with weak SFA which cannot stabilize run-away activity. Different colors represent different adaptation strengths $b$. (B) Characteristic function $F(z)$ in networks with strong SFA capable of generating a limit cycle. (C) Firing rates of the excitatory (blue) and inhibitory population (red) in the presence of strong SFA (left). The zoomed-in activity from $3.0 \mathrm{~s}$ to $3.2 \mathrm{~s}$ (right) corresponding to the green period (left) indicates oscillatory behavior in networks with strong SFA.
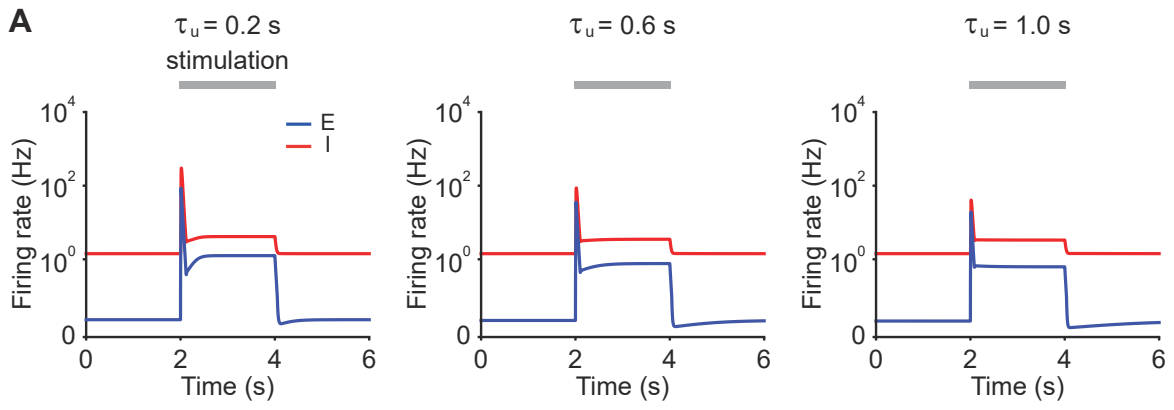

B
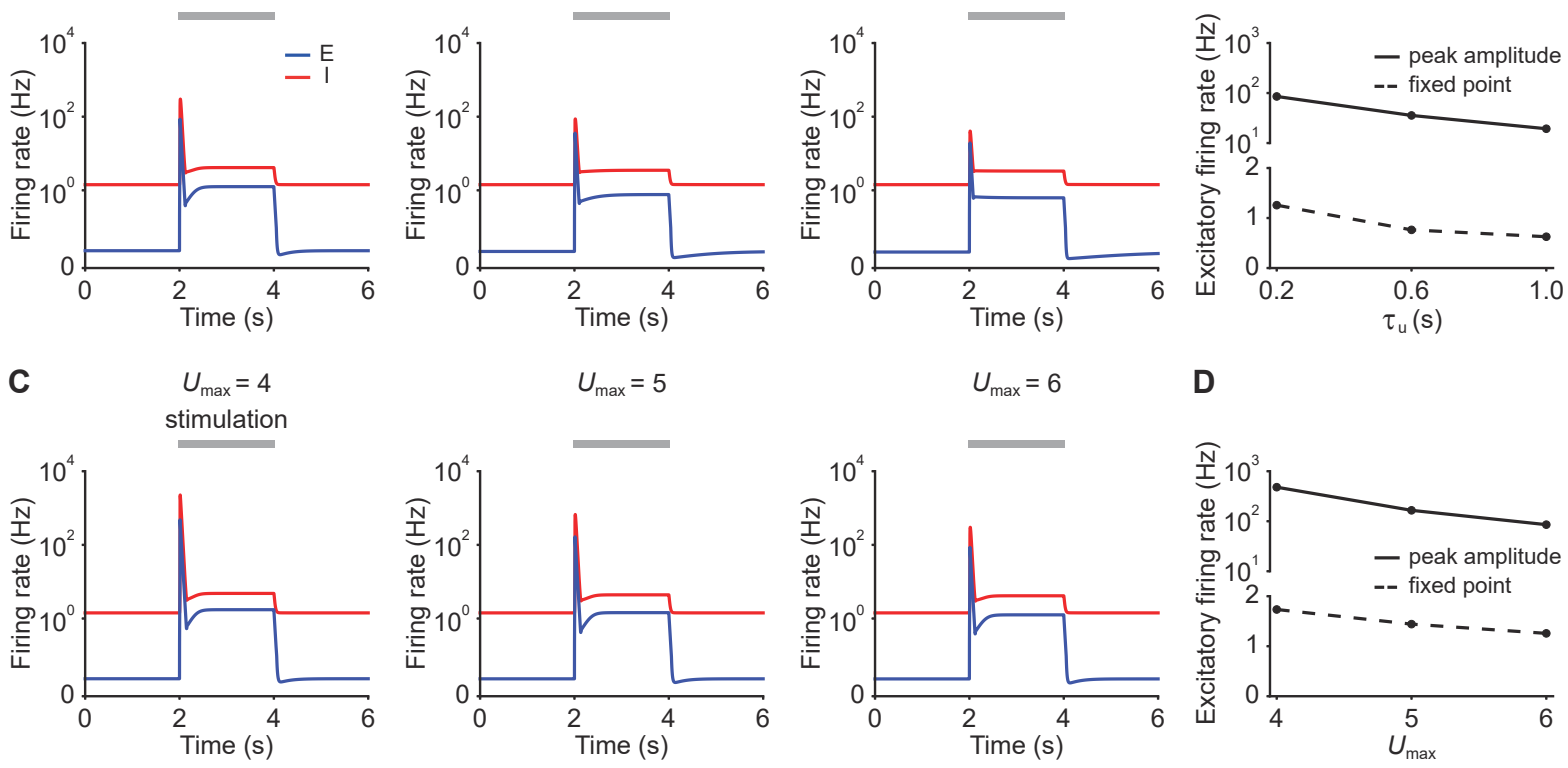

D
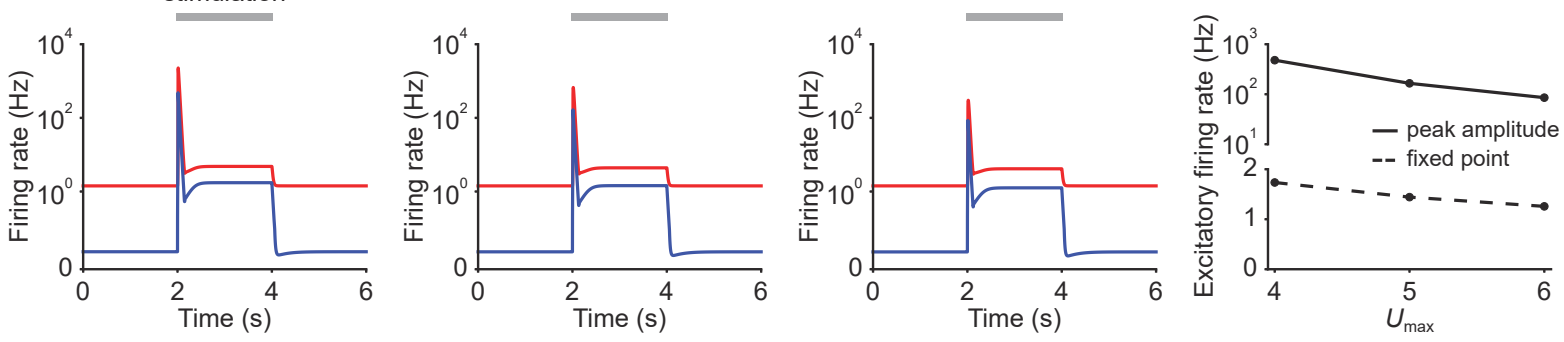

Fig. S2. (A) Firing rates of the excitatory (blue) and inhibitory population (red) in response to stimulation in the presence of E-to-I STF with different time constants $\tau_{u}$. The stimulation period from $2 \mathrm{~s}$ to $4 \mathrm{~s}$ is marked with the gray bar. The stimulation is implemented by changing input $g_{E}$. (B) Peak amplitude (solid line) and fixed-point activity (dashed line) of the excitatory population during stimulation with different STF time constants. (C and D) Same as A and B, but with different maximum allowed facilitation levels $U_{\max }$. 

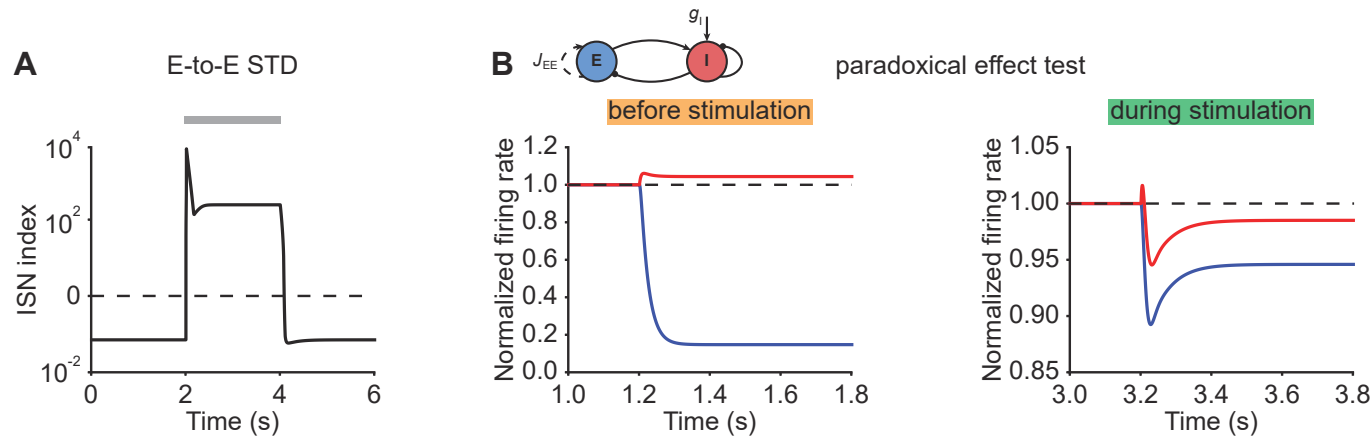

Fig. S3. (A) ISN index of the network with E-to-E STD in Fig. 2B (left). The horizontal dashed line indicates an ISN index of 0 . (B) The normalized firing rates of the excitatory (blue) and inhibitory population (red) when injecting excitatory input into the inhibitory population of an active ensemble (cf. Fig. 2B) starting at $1.2 \mathrm{~s}$ while the ensemble receives external stimulation (left), and at $3.2 \mathrm{~s}$ (right). The horizontal dashed lines indicate a normalized firing rate of 1.0.

A

naive rate-based model stimulation

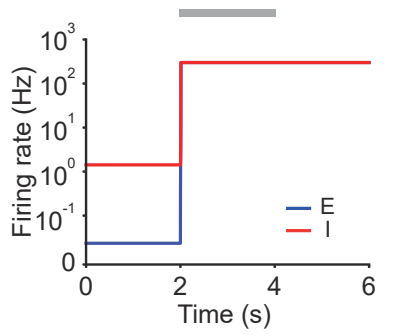

B spike-frequency adaptation

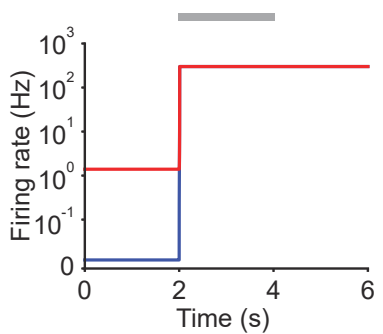

C

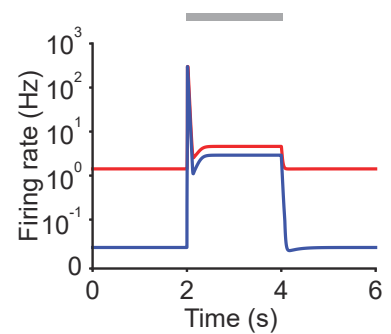

D E-to-I STF

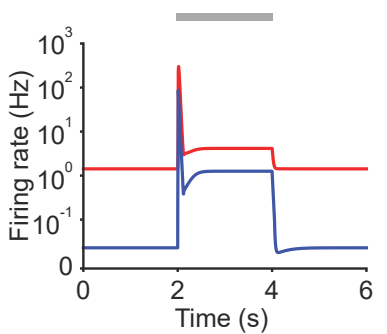

E

strong spike-frequency adaptation
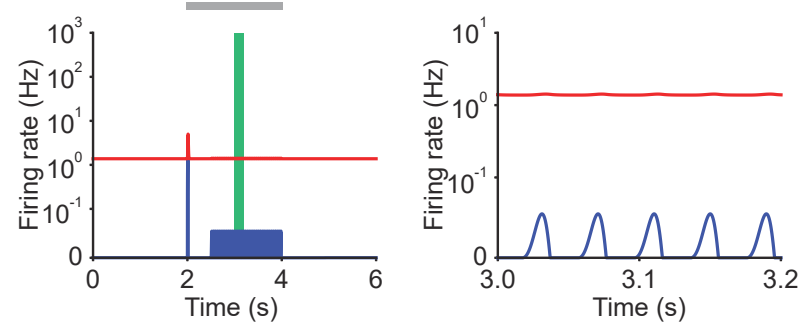

Fig. S4. (A) Firing rates of the excitatory (blue) and inhibitory population (red) in response to stimulation in a rate-based model. Additional excitatory inputs are injected into excitatory neurons in the period marked in gray. Firing rates are capped at $300 \mathrm{~Hz}$. (B) Same as A but incorporating SFA. (C) Same as A but incorporating E-to-E STD. (D) Same as A but incorporating E-to-I STF. (E) Same as B but incorporating stronger SFA (left). The zoomed-in activity from $3.0 \mathrm{~s}$ to $3.2 \mathrm{~s}$ (right) corresponding to the green period (left) indicates oscillatory behavior in networks with strong SFA. 
bioRxiv preprint doi: https://doi.org/10.1101/2021.06.09.447718; this version posted June 10,2021. The copyright holder for this preprint (which was not certified by peer review) is the author/funder, who has granted bioRxiv a license to display the preprint in perpetuity. It is made available under aCC-BY 4.0 International license.

A naive spiking neural network $\mathbf{B}$ spike-frequency adaptation

C stimulation
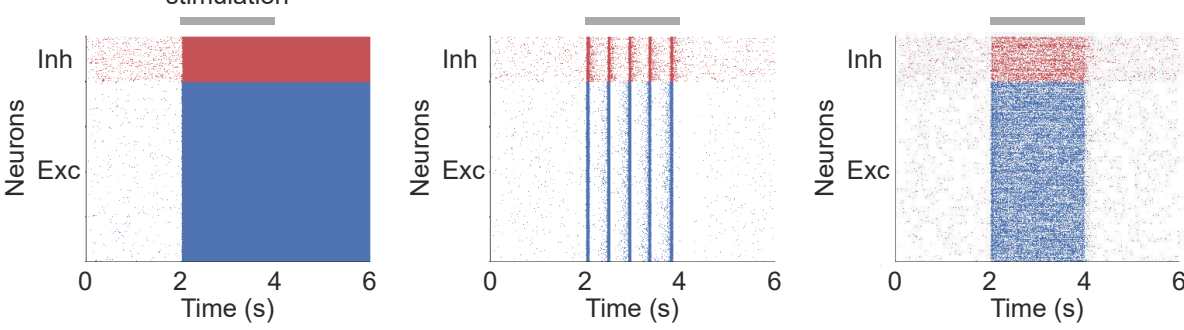

D E-to-I STF
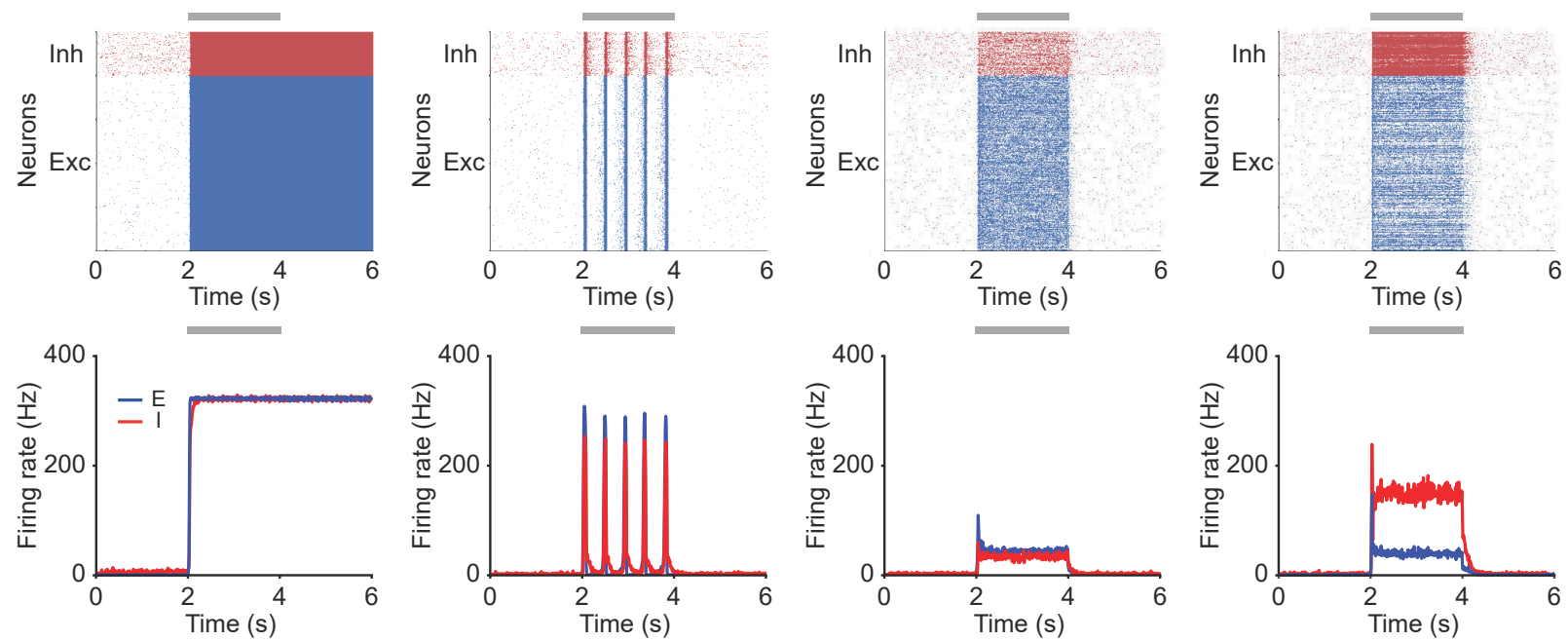

Fig. S5. (A) Spike raster plot of the excitatory (top, blue) and inhibitory population (top, red) in response to stimulation in a spiking neural network model, and firing rates calculated with $10 \mathrm{~ms}$ time bins (bottom). Additional excitatory inputs are injected into excitatory neurons in the period marked in gray. (B) Same as A but incorporating SFA. (C) Same as A but incorporating E-to-E STD. (D) Same as A but incorporating E-to-I STF.

A

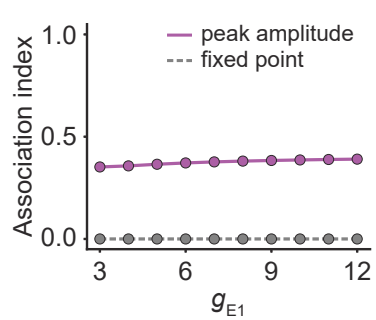

B

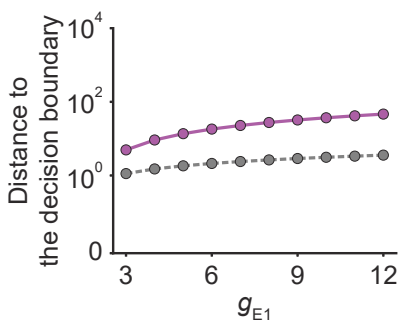

C

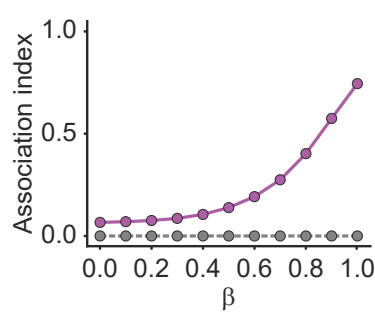

D

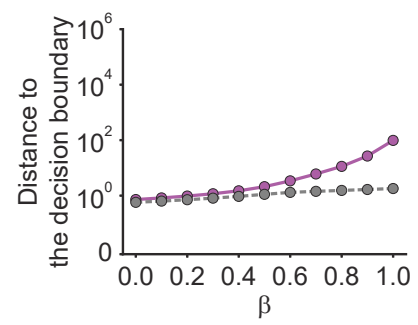

Fig. S6. (A) Association index as a function of input $g_{E 1}$ for the onset peak amplitude (magenta solid line) and fixedpoint activity (gray dashed line) for E-to-I STF. (B) Distance to the decision boundary as a function of input $g_{E 1}$ for the onset peak amplitude (magenta solid line) and fixed-point activity (gray dashed line) for E-to-I STF. (C and D) Same as $\mathrm{A}$ and $\mathrm{B}$ but as a function of $\beta$, which controls the inner- and inter-ensemble connection strength.
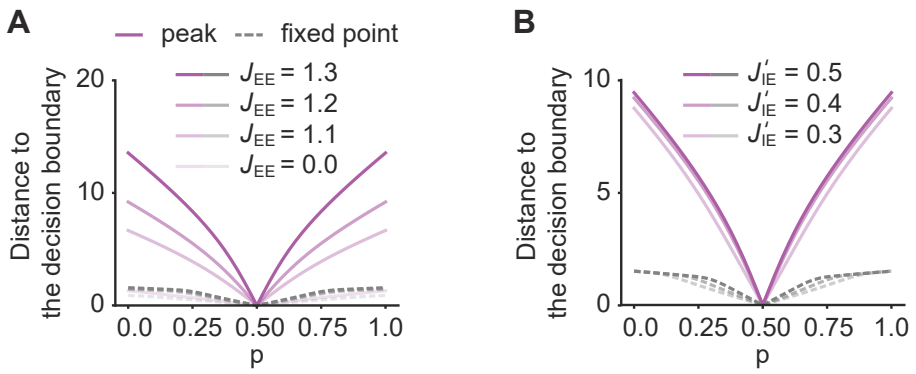

Fig. S7. (A) Distance to the decision boundary as a function of $p$ for the onset peak amplitude (magenta solid lines) and fixed-point activity (gray dashed lines) for E-to-I STF. Different levels of brightness represent different recurrent E-to-E connection strengths $J_{E E}$. (B) Same as A but with different E-to-I connection across ensembles strengths $J_{I E}^{\prime}$. 


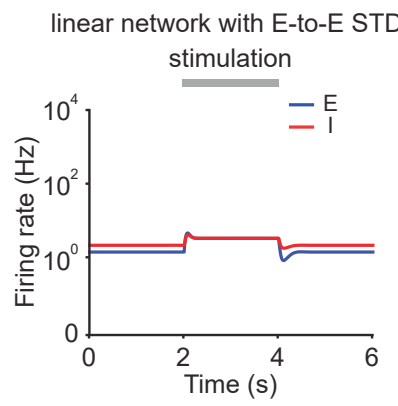

Fig. S8. Firing rates of the excitatory (blue) and inhibitory population (red) in linear networks with E-to-E STD. During stimulation (gray bar) additional input is injected into the excitatory population. Same as Fig. $2 \mathrm{~B}$ (left) but with $\alpha_{E}=\alpha_{l}=$ 1.

A

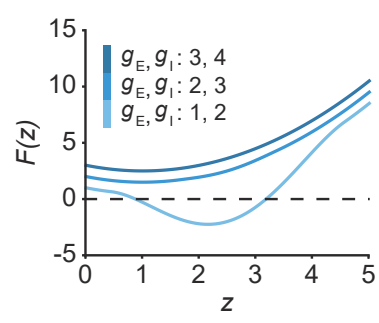

B

I $g_{\mathrm{E}}, g_{1}: 1,2$

stimulation

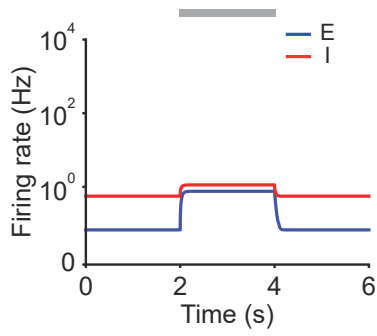

$g_{\mathrm{E}}, g_{1}: 2,3$

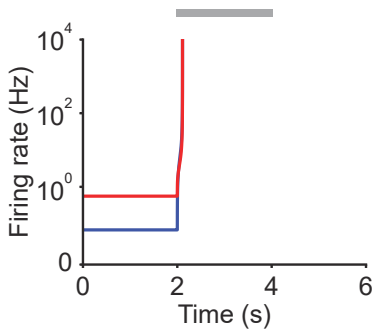

$g_{\mathrm{E}}, g_{1}: 3,4$

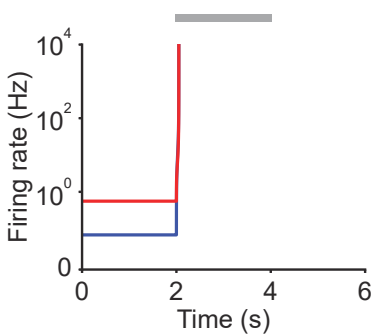

Fig. S9. (A) Characteristic function $F(z)$ for different inputs $g_{E}$ and $g_{l}$. (B) Firing rates of the excitatory (blue) and inhibitory population (red) in response to stimulation from $2-4 \mathrm{~s}$ (gray bar). During stimulation $g_{E}$ and $g_{l}$ are simultaneously changed to the stated values. 